\title{
Dynamic Subgrid Scale Modeling of Turbulent Flows using Lattice-Boltzmann Method
}

\author{
Kannan N. Premnath ${ }^{\mathrm{a}, \mathrm{b}, *}$ Martin J. Pattison $^{\mathrm{a}}$ \\ Sanjoy Banerjee ${ }^{a, b, c, d}$ \\ ${ }^{a}$ MetaHeuristics LLC, 3944 State Street, Suite 350, Santa Barbara, CA 93105 \\ ${ }^{\mathrm{b}}$ Department of Chemical Engineering, University of California Santa Barbara, \\ Santa Barbara, CA 93106 \\ ${ }^{\mathrm{c}}$ Department of Mechanical Engineering, University of California Santa Barbara, \\ Santa Barbara, CA 93106 \\ ${ }^{\mathrm{d}}$ Bren School of Environmental Science and Management, University of California \\ Santa Barbara, Santa Barbara, CA 93106
}

\begin{abstract}
In this paper, we discuss the incorporation of dynamic subgrid scale (SGS) models in the lattice-Boltzmann method (LBM) for large-eddy simulation (LES) of turbulent flows. The use of a dynamic procedure, which involves sampling or test-filtering of super-grid turbulence dynamics and subsequent use of scale-invariance for two levels, circumvents the need for empiricism in determining the magnitude of the model coefficient of the SGS models. We employ the multiple relaxation times (MRT) formulation of LBM with a forcing term, which has improved physical fidelity and numerical stability achieved by proper separation of relaxation time scales of hydrodynamic and non-hydrodynamic modes, for simulation of the grid-filtered dynamics of large-eddies. The dynamic procedure is illustrated for use with the common Smagorinsky eddy-viscosity SGS model, and incorporated in the LBM kinetic approach through effective relaxation time scales. The strain rate tensor in the SGS model is locally computed by means of non-equilibrium moments of the MRT-LBM. We also discuss proper sampling techniques or test-filters that facilitate implementation of dynamic models in the LBM. For accommodating variable resolutions, we employ conservative, locally refined grids in this framework. As examples, we consider the canonical anisotropic and inhomogeneous turbulent flow problem, i.e. fully developed turbulent channel flow at two different shear Reynolds numbers $R e_{*}$ of 180 and 395 . The approach is able to automatically and self-consistently compute the values of the Smagorinsky coefficient, $C_{S}$. In particular, the computed value in the outer or bulk flow region, where turbulence is generally more isotropic, is about 0.155 (or the model coefficient $C=C_{S}^{2}=0.024$ ) which is in good agreement with prior data. It is also shown that the model coefficient becomes smaller and
\end{abstract}

Preprint submitted to Elsevier 31 October 2018 
approaches towards zero near walls, reflecting the dampening of turbulent length scales near walls. The computed turbulence statistics at these Reynolds numbers are also in good agreement with prior data. The paper also discusses a procedure for incorporation of more general scale-similarity based SGS stress models.

Key words: Lattice-Boltzmann Method, Multiple-Relaxation-Time Model, Turbulent Flows, Large Eddy Simulation, Dynamic Subgrid Scale Modeling , 47.11.+j, 05.20.Dd, 47.65.Md

\section{Introduction}

Turbulence in fluids is ubiquitous in nature and technological systems and represents one of the most challenging aspects in fluid mechanics. The difficulty stems from the inherent presence of many scales that are generally inseparable, among many other factors. Nevertheless, considerable progress has been made over the years towards more fundamental physical understanding of turbulence phenomena through measurements, statistical phenomenological theories, modeling and computation [1,2,3].

In the case of their modeling and computation, different approaches, depending on the desired degree of detail, have been devised. One approach is to employ Reynolds-averaged equations of fluid motion representing the evolution of mean turbulence field. In this framework, the averaged turbulent fluctuations - the Reynolds stresses - need to be modeled. Various turbulence models have been developed for this purpose, including the well-known $k-\epsilon$ and Reynolds stress transport equations. A major limitation of this approach is that Reynolds-averaged models need to represent physics over a wide range of scales. While small scale turbulence tends be somewhat more universal, large scale turbulent motions are strongly problem dependent. Thus, it is unrealistic to expect the Reynolds-averaged models to accommodate and represent large-scale behavior of different classes of turbulent flows in the same manner without resorting to considerable empiricism.

On the other hand, direct numerical simulation (DNS) approach resolves all spatio-temporal scales up to dissipation scales without the use of models, and can thus predict all possible motions, structural and statistical features due to turbulence with high fidelity. Due to its high computational cost, its application is restricted to relatively low Reynolds numbers. On the other hand, in

\footnotetext{
* Corresponding author.

Email address: nandha@metah.com (Kannan N. Premnath).
} 
view of this, it is more pragmatic to only compute the eddying motions of fluids with length scales greater than the grid size and model unresolved subgrid scales (SGS), which comprises the large eddy simulation (LES) approach. The SGS scales, which mainly account for dissipation of turbulence, are generally more isotropic in nature and relatively independent of the resolved large-scale fluid dynamics. Thus, LES represents a compromise with reduced empiricism in contrast to Reynolds-averaged models, but with reduced computational cost in comparison with DNS.

In LES approach, the Smagorinsky eddy-viscosity model (1963) [4] is one of the simplest SGS models. Based on the assumption that the small scales are in equilibrium, they dissipate all the energy transferred from large scales instantaneously and completely. This dissipation mechanism is modeled by an eddy viscosity, which is represented by the product of the square of a length scale, which is often the grid size, and the inverse of a time scale, which is given by the local fluid shear rate, with a proportionality coefficient or "constant". The values of this proportionality "constant" were first determined by Lilly (1966) [5] and Deardorff (1970) [6] by analysis for isotropic turbulence and turbulent channel flow, respectively, and one of the first successful LES applications is by Schumann (1975) [7]. A more theoretical basis for LES was first provided by Leonard (1974) [8] through the introduction of formal filtering procedures on fluctuating turbulence fields.

In wall-bounded flows, turbulence becomes anisotropic close to walls, with its length scales becoming progressively smaller and vanishing at the wall. Thus, with the use of a finite grid size as the length scale in the SGS model, the proportionality coefficient should decrease and approach to zero towards the wall to account for near-wall turbulence anisotropy and the model coefficient is actually not a "constant". Often, an ad hoc damping function [9] is used for this purpose [10]. Moreover, in transitional flows, it is found the use of the "constant" Smagorinsky SGS model in LES can cause excessive damping of the resolved structures [11] and can be alleviated by an additional ad hoc intermittency function. The need to a priori specify the values of the proportionality coefficient and their variation, for example, in the presence of turbulence anisotropy or laminar-to-turbulence transition comprises the empirical elements of the LES approach.

Significant progress in the LES approach was made by the introduction of dynamic procedures for local computation of the coefficient(s) in the SGS models by Germano and co-workers [12,13]. By obtaining information of the turbulence field at scales larger than the grid size - the "test" filter scale, and relating them with the resolved turbulence at grid scale through scaleinvariance, the value of the model coefficient can be computed dynamically as the computation progresses. Thus, dynamic procedures avoid the need to a priori specify the model coefficient and determine them based on the local 
fluctuating turbulence field. Moreover, unlike the constant Smagorinsky SGS model, it does not rule out the possibility of backscattering of energy from smaller scales. It is found that the use of dynamic procedure makes LES susceptible to numerical instabilities, which can be substantially alleviated by employing a least squares technique [14 in conjunction with the use of averaging to compute the coefficient.

When the dynamic procedure is applied to the Smagorinsky SGS model, it is often simply referred to as the dynamic Smagorinsky model (DSM). Other SGS models include the use of a scale-similarity stress tensor combined with the Smagorinksy model - the mixed SGS model [15] - to provide improved predictions for certain classes of inhomogeneous flows. Dynamic procedures have been applied with much success to mixed SGS models, resulting in different variants such as the dynamic mixed model (DMM) [16] and the dynamic two-parameter model (DTM) [17. The remarkable progress made in the development of LES as a technique for turbulence simulation and their applications have been discussed in detail in the reviews by Piomelli (1999) [18], Meneveau and Katz (2000) [19] and Pope (2004) [20, and in the monographs of Galperin and Orszag (1993) [21] and Sagaut (2003) [22.

In this paper, we propose to incorporate dynamic procedure in the lattice Boltzmann method (LBM) for LES. In recent years, the LBM, based on minimal discrete kinetic models, has emerged as an alternative and accurate computational approach for fluid mechanics problems [23,24]. It involves the solution of the lattice-Boltzmann equation (LBE) that represents the changes in the evolution of the distribution of particle populations due to their advection, represented as a free-flight process, and collision, described as a relaxation process, on a lattice. When the lattice, which represents the discrete directions for particle propagation, satisfies sufficient rotational symmetries, the averaged LBE asymptotically recovers the weakly compressible Navier-Stokes equations (NSE).

Though it evolved as a computationally efficient form of lattice gas cellular automata [25], it was well established about a decade ago that the LBE is actually a much simplified form of the Boltzmann equation [26]27]. As a result, several previous results in discrete kinetic theory could be directly applied to the LBE. This lead to, for example, improved physical modeling in various situations, such as multiphase flows [28,29] and multicomponent flows [30], and in an asymptotic theory suitable for rigorous numerical analysis [31]. As a result of features of the stream-and-collide procedure of the LBE such as the algorithmic simplicity, amenability to parallelization with near-linear scalability, and its ability to represent complex boundary conditions and incorporate physical models more naturally, it has rapidly found a wide range of applications $32,33,34,35$. 
Discrete kinetic theory, and in particular the LBM, has recently been employed to a priori derive turbulence models based on a mean-field approximation [36,37,38] and lattice kinetic turbulence models have found much success [39]. It has now been well established that LBM is a reliable and accurate method for direct numerical simulation (DNS) of various benchmark turbulent flow problems [40,41,42,43,44]. On the other hand, in earlier work, the use of LBM as a LES tool with "constant" Smagorinsky model to represent SGS effects was also proposed [45,46], which has more recently found applications for flows in different configurations and physical conditions [47,48,49,50].

A commonly used form of the LBM employs a single relaxation time (SRT) model [51] to represent the effect of particle collisions, in which particle distributions relax to their local equilibrium at a rate determined by a single parameter [52,53]. On the other hand, an equivalent representation of distribution functions is in terms of their moments, such as various hydrodynamic fields including density, mass flux, and stress tensor. The relaxation process due to collisions can more naturally be described in terms of a space spanned by such moments, which can, in general relax at different rates. This forms the basis of the generalized lattice-Boltzmann equation (GLBE) based on multiple relaxation times (MRT) [54,55,56].

By carefully separating the time scales of various hydrodynamic and kinetic modes through a linear stability analysis, the numerical stability of the GLBE or MRT-LBE can be significantly improved when compared with the SRTLBE, particularly for more demanding problems at high Reynolds numbers [55]. MRT-LBE has also been extended for multiphase flows with superior stability characteristics [57,58,59,60], and, more recently, for magnetohydrodynamic problems [61]. It has also been used for LES of a class of turbulent flows [48,50]. Recently, we have extended the GLBE with forcing terms for wall-bounded turbulent flows 62, which is a generalization of the approach introduced earlier by $\mathrm{Yu}$ et al. [50]. These forcing terms, determined to avoid discrete lattice artifacts, represent the effect of external forces and are considered in natural moment space of GLBE. In this work, we represented the SGS effects through the relaxation times of the hydrodynamic moments using the "constant" Smagorinsky eddy-viscosity model [4], which is modified by the van Driest damping function [9] in the near wall region.

One of the main objectives of this paper is to develop and implement a framework for application of dynamic procedure in the lattice Boltzmann method (LBM) for LES of inhomogeneous and anisotropic turbulent flows. In view of the previous discussions, we plan to employ GLBE based on multiple relaxation times as the basis for this purpose. We will present the incorporation of the dynamic procedure for the Smagorinsky SGS model, i.e. DSM, in LBM. Particular attention is paid to performing discrete filtering operations at super lattice grid or test-filter levels, which will be discussed. To accommodate 
efficient simulation of varying turbulent length scales in the near-wall region, variable resolution strategies are required. In this regard, we employ conservative, locally refined multiblock grids that allow variable resolution. The approach will be tested for the fully-developed turbulent channel flow problem at two different shear Reynolds numbers $R e_{*}$ of 180 and 395, by comparing the turbulent statistics with prior data based on direct numerical simulations (DNS) and measurements. The higher Reynolds number case, to the best of our knowledge, is considered for the first time in the LBM for LES of turbulent channel flows. The incorporation of DSM in the GLBE is a first step in the application of dynamic procedure. More general SGS models, involving scale-similarity stresses, such as DMM and DTM can also be introduced in the GLBE, which will also be briefly described in an appendix of this paper, and their implementation will be a subject of future work.

The organization of this paper is as follows: In Sec. 2, we will discuss the generalized lattice-Boltzmann equation (GLBE) with forcing term. The use of conservative locally refined multiblock grids will be presented in Sec. 3. Section 4 will discuss the dynamic SGS modeling with DSM in GLBE framework. Then, Sec. [5 will present the LES results for the canonical turbulent channel flow problem at two different Reynolds numbers. Finally, the summary and conclusions of this paper will be presented in Sec. 6 .

\section{Generalized Lattice Boltzmann Equation with Forcing Term}

We shall now discuss the computational procedure based on generalized latticeBoltzmann equation with a forcing term, which is supplemented by Smagorinsky SGS model with a variable coefficient, obtained by means of a dynamic procedure (see next section). For brevity, we will present only the major elements of the approach, while the details can be found in Refs. [56,50,58,62].

The lattice-Boltzmann method computes the evolution of distribution functions as they move and collide on a lattice grid. The collision process consider their relaxation to their local equilibrium values, and the streaming process describes their movement along the characteristics directions given by a discrete particle velocity space represented by a lattice. Figure 1 represents the three-dimensional, nineteen particle velocity (D3Q19) lattice model employed in this paper. The particle velocity $\overrightarrow{e_{\alpha}}$ corresponding to this model may be written as:

$$
\overrightarrow{e_{\alpha}}= \begin{cases}(0,0,0) & \alpha=0 \\ ( \pm 1,0,0),(0, \pm 1,0),(0,0, \pm 1) & \alpha=1, \cdots, 6 \\ ( \pm 1, \pm 1,0),( \pm 1,0, \pm 1),(0, \pm 1, \pm 1) & \alpha=7, \cdots, 18\end{cases}
$$




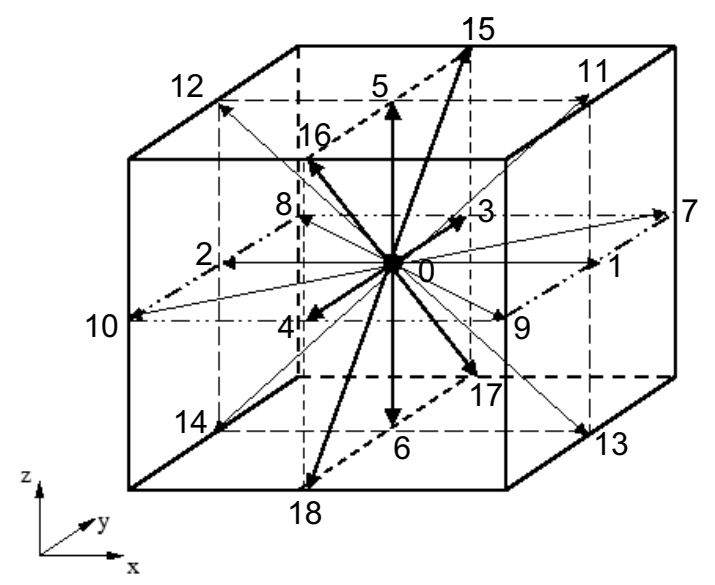

Fig. 1. Schematic illustration of the three-dimensional, nineteen velocity (D3Q19) model.

The GLBE computes collision in moment space, while the streaming process is performed in the usual particle velocity space [56]. The GLBE with forcing term [62] also computes the forcing term, which represents the effect of external forces as a second-order accurate time-discretization, in moment space. We use the following notation in our description of the procedure below: In particle velocity space, the local distribution function $\mathbf{f}$, its local equilibrium distribution $\mathbf{f}^{e q}$, and the source terms due to external forces $\mathbf{S}$ may be written as the following column vectors: $\mathbf{f}=\left[f_{0}, f_{1}, f_{2}, \ldots, f_{18}\right]^{\dagger}, \mathbf{f}^{e q}=\left[f_{0}^{e q}, f_{1}^{e q}, f_{2}^{e q}, \ldots, f_{18}^{e q}\right]^{\dagger}$, and $\mathbf{S}=\left[S_{0}, S_{1}, S_{2}, \ldots, S_{18}\right]^{\dagger}$. Here, the superscript $\dagger$ represents the transpose operator.

The moments $\widehat{\mathbf{f}}$ are related to the distribution function $\mathbf{f}$ through the relation $\widehat{\mathbf{f}}=\mathcal{T} \mathbf{f}$ where $\mathcal{T}$ is the transformation matrix. Here, and in the following, the "hat" represents the moment space. The transformation matrix $\mathcal{T}$ is constructed such that the collision matrix in moment space $\widehat{\Lambda}$ is a diagonal matrix through $\widehat{\Lambda}=\mathcal{T} \Lambda \mathcal{T}^{-1}$, where $\Lambda$ is the collision matrix in particle velocity space. The elements of $\mathcal{T}$ are obtained in a suitable orthogonal basis as combinations of monomials of the Cartesian components of the particle velocity $\overrightarrow{e_{\alpha}}$ through the standard Gram-Schmidt procedure, which are provided by d'Humières et al. (2002) [56]. Similarly, the equilibrium moments and the source terms in moment space may be obtained through the transformation $\widehat{\mathbf{f}}^{e q}=\mathcal{T} \mathbf{f}^{e q}, \widehat{\mathbf{S}}=\mathcal{T} \mathbf{S}$. The components of moment-projections of these quantities are: $\widehat{\mathbf{f}}=\left[\widehat{f}_{0}, \widehat{f}_{1}, \widehat{f}_{2}, \ldots, \widehat{f}_{18}\right]^{\dagger}, \widehat{\mathbf{f}}^{e q}=\left[\hat{f}_{0}^{e q}, \widehat{f}_{1}^{e q}, \hat{f}_{2}^{e q}, \ldots, \widehat{f}_{18}^{e q}\right]^{\dagger}$, and $\widehat{\mathbf{S}}=\left[\widehat{S}_{0}, \widehat{S}_{1}, \widehat{S}_{2}, \ldots, \widehat{S}_{18}\right]^{\dagger}$. These are provided in Appendix $\mathrm{A}$.

The solution of the GLBE with forcing term can be written in terms of the 
following "effective" collision and streaming steps, respectively:

$$
\widetilde{\mathbf{f}}(\vec{x}, t)=\mathbf{f}(\vec{x}, t)+\varpi(\vec{x}, t)
$$

and

$$
f_{\alpha}\left(\vec{x}+\vec{e}_{\alpha} \delta_{t}, t+\delta_{t}\right)=\tilde{f}_{\alpha}(\vec{x}, t)
$$

where the distribution function $\mathbf{f}=\left\{f_{\alpha}\right\}_{\alpha=0,1, \ldots, 18}$ is updated due to "effective" collision resulting in the post-collision distribution function $\widetilde{\mathbf{f}}=\left\{\widetilde{f}_{\alpha}\right\}_{\alpha=0,1, \ldots, 18}$ before being shifted along the characteristic directions during streaming step. $\varpi$ represents the change in distribution function due to collisions as a relaxation process and external forces, and following Premnath et al. (2008) [62] it can written as

$$
\varpi(\vec{x}, t)=\mathcal{T}^{-1}\left[-\widehat{\Lambda}\left(\widehat{\mathbf{f}}-\widehat{\mathbf{f}}^{e q}\right)_{(\vec{x}, t)}+\left(\mathcal{I}-\frac{1}{2} \widehat{\Lambda}\right) \widehat{\mathbf{S}}_{(\vec{x}, t)}\right]
$$

where $\mathcal{I}$ is the identity matrix and $\widehat{\Lambda}=\operatorname{diag}\left(s_{0}, s_{1}, \ldots, s_{18}\right)$ is the diagonal collision matrix in moment space. Now, some of the relaxation times $s_{\alpha}$ in the collision matrix, i.e. those corresponding to hydrodynamic modes can be related to the transport coefficients and modulated by eddy-viscosity due to SGS model (discussed below) as follows [56,50,62]: $s_{1}^{-1}=\frac{9}{2} \zeta+\frac{1}{2}$, where $\zeta$ is the molecular bulk viscosity, and $s_{9}=s_{11}=s_{13}=s_{14}=s_{15}=s_{\nu}$, where $s_{\nu}^{-1}=3 \nu+\frac{1}{2}=3\left(\nu_{0}+\nu_{t}\right)+\frac{1}{2}$, with $\nu_{0}$ being molecular shear viscosity and $\nu_{t}$ the eddy-viscosity determined from the dynamic SGS model discussed in the next section. The rest of the relaxation parameters, i.e. for the kinetic modes, can be chosen through a von Neumann stability analysis of the linearized GLBE [56]: $s_{1}=1.19, s_{2}=s_{10}=s_{12}=1.4, s_{4}=s_{6}=s_{8}=1.2$ and $s_{16}=s_{17}=s_{18}=1.98$.

Once the distribution function is known, the hydrodynamic fields, i.e., the density $\rho$, velocity $\vec{u}$ and pressure $p$ can be obtained as follows:

$$
\rho=\sum_{\alpha=0}^{18} f_{\alpha}, \quad \vec{j} \equiv \rho \vec{u}=\sum_{\alpha=0}^{18} f_{\alpha} \vec{e}_{\alpha}+\frac{1}{2} \vec{F} \delta_{t}, \quad p=c_{s}^{2} \rho,
$$

where, $c_{s}=c / \sqrt{3}$ with $c=\delta_{x} / \delta_{t}$ being the particle speed, and $\delta_{x}$ and $\delta_{t}$ are the lattice spacing and time step, respectively. It can be readily shown that when a multiscale analysis based on the Chapman-Enskog expansion [63,58] is applied to the GLBE with relaxation times scales augmented by an eddy-viscosity, it recovers the "grid filtered" weakly compressible Navier-Stokes equations with external forces. That is, the density, momentum and pressure obtained from Eq. (5) are grid-filtered quantities: $\rho \rightarrow \bar{\rho}, \rho \vec{u} \rightarrow \bar{\rho} \vec{u}$ and $p \rightarrow \bar{p}$, where 
the 'overbar' represents grid-filter. It may be noted that the macrodynamical equations can alternatively be derived through an asymptotic analysis under a diffusive scaling [31.

The computational procedure for the solution of the GLBE with forcing term is optimized by fully exploiting the special properties of the transformation matrix $\mathcal{T}$ : these include its orthogonality, entries with many zero elements, and entries with many common elements that are integers, which are used to form the most common sub-expressions for transformation between spaces in avoiding direct matrix multiplications [56. For details, we refer the reader to Ref. 5062 . As a result of such optimization, the additional computational overhead when GLBE is used in lieu of the popular SRT-LBE is small, typically between $15 \%-30 \%$, but with much enhanced numerical stability that allows maintaining solution fidelity on coarser grids and also in simulating flows at higher Reynolds numbers.

\section{Conservative Local Grid Refinement Based on Multiblock Ap- proach}

Closer to a wall, length scales are very small, requiring a fine grid to adequately resolve turbulent structures. Use of grid fine enough to resolve the wall regions throughout the domain can entail significant computational cost, and this can be mitigated by introducing coarser grids farther from the wall, where turbulent length scales are larger. One approach is to consider using continuously varying grid resolutions, using a interpolated-supplemented LBM [64] that effectively decouples particle velocity space represented by the lattice and the computational grid. However, it is well known that interpolation could introduce significant numerical dissipation, see for e.g. [55, which could severely affect the accuracy of solutions involving turbulent fluctuations. Thus, we consider locally embedded grid refinement approaches, and in particular their conservative versions [65, 66 that preserve mass and momentum conservation. Similar zonal embedded approaches have been successfully employed in computational approaches based on the solution of filtered NSE for LES of turbulent flows [67].

Figure 2 shows a schematic of such a multiblock approach in which a fine cubic lattice grid is used close to the bottom wall surfaces and a coarser one, again cubic in shape, farther out. Similarly, finer grids are employed near top free-slip surfaces. In order to facilitate the exchange of information at the interface between the grids, the spacing of the nodes changes by an integer factor, in this case two. As well as using different grid sizes, the two regions use different time steps (time step being proportional to grid size), and the computational cost required per unit volume is thus reduced by a factor of 


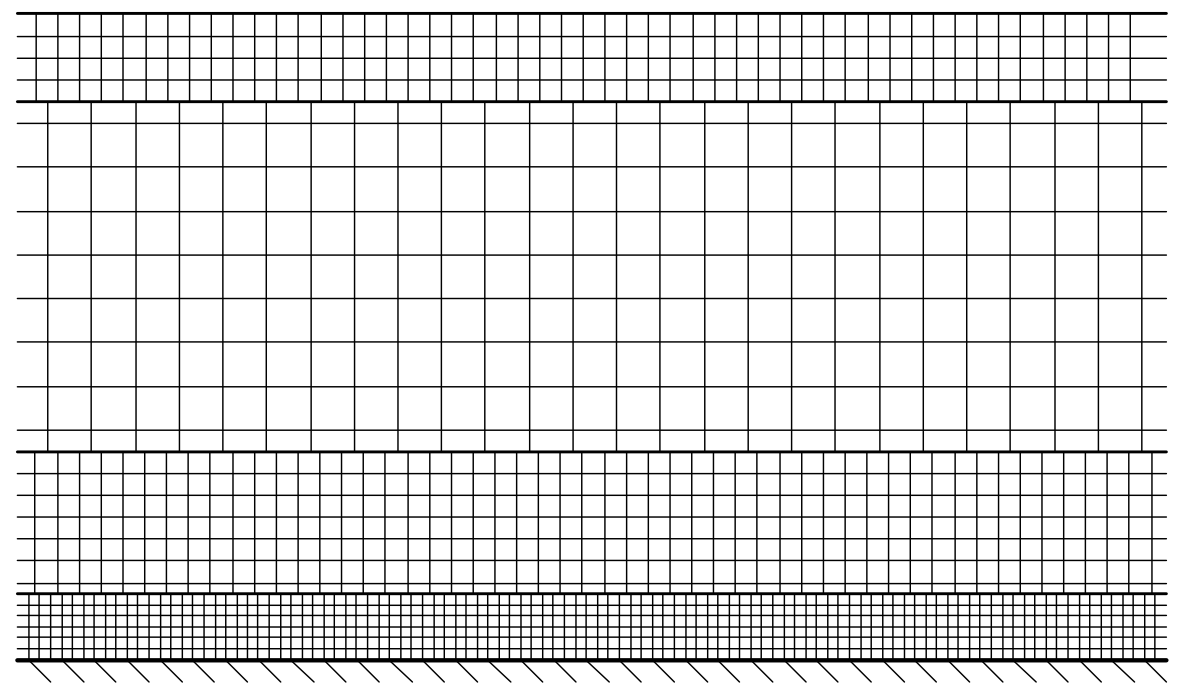

Fig. 2. Schematic illustration of conservative, locally refined multiblock gridding strategy for LES of turbulent channel flow using GLBE with dynamic SGS model.

16 in the coarse grid. Figure 2 shows a staggered grid arrangement, in which nodes on the fine and coarse sides of the interface are arranged in a manner that facilitates the imposition of mass and momentum conservation. Different blocks communicate with each other through the Coalesce and the Explode steps, in addition to the standard stream-and-collide procedure. The details are provided in Refs. [65,66] and we very briefly present the essential elements in the following.

The Coalesce procedure involves summing the particle populations on the fine nodes to provide new incoming particle populations for the corresponding coarse nodes. Similarly, the Explode step involves redistributing the populations on the coarse node to the surrounding fine nodes. These grid - communicating steps used in the multiblock approach presented in Ref. [65] were incorporated in GLBE framework in this work. It may be noted that other approaches to local grid refinement exist in the literature. In particular, asymptotic analysis of the LBE shows that it converges to the incompressible NSE when the ratio of the square of time step and square of the grid spacing is a constant [31]. Local grid refinement based on this consideration need to 
adapt the time step to vary as the square of the grid spacing. However, in this work we use the grid refinement variant that considers linear variation of the time step with the grid spacing for reasons of relatively lower computational cost and the fact that it is already more developed and tested for different problems 6566].

\section{Subgrid Scale Eddy-Viscosity Modeling using Dynamic Proce- dure}

The main idea behind the application of dynamic procedure to SGS models [12] is that the local value of the model coefficient of the eddy viscosity, representing eddy-viscosity type subgrid scale effects, can be obtained from sampling the smallest super-grid or resolved scales, which are generally referred to as the test-filtered scales, and assuming scale-invariance at these two levels. If $\bar{\Delta}$ is the width of the grid filter, which in the GLBE becomes $\bar{\Delta}=\delta_{x}$, where $\delta_{x}$ is the lattice grid spacing, the flow information is sampled at a larger scale $\tilde{\bar{\Delta}}$, the test-filter width, i.e. $\widetilde{\bar{\Delta}}>\bar{\Delta}$, and generally $\tilde{\bar{\Delta}} / \bar{\Delta}=2[12]$. The notation adapted here, as before, and in the following is that 'bar' refers to grid-filtered values and a 'tilde' refers to test-filtered values. The effect of subgrid scales is parameterized by an eddy viscosity relation [4]

$$
\nu_{t}=C \bar{\Delta}^{2}|\bar{S}|
$$

where $|\bar{S}|$ is the strain rate, given by $|\bar{S}|=\sqrt{2 \bar{S}_{i j} \bar{S}_{i j}}$ and $C$ is the model coefficient. The grid-filtered strain rate $|\bar{S}|$ can be obtained by evaluating the corresponding strain rate tensor from the non-equilibrium moments as shown in Appendix A. The model coefficient $C$ is obtained as follows.

The anisotropic part of the SGS stress at grid-filter scale $\tau_{i j}\left(\tau_{i j}=\bar{u}_{i} \bar{u}_{j}-\overline{u_{i} u_{j}}\right)$ and that at the test-filter scale $T_{i j}\left(T_{i j}=\widetilde{\bar{u}}_{i} \widetilde{\bar{u}}_{j}-\widetilde{u_{i} u_{j}}\right)$, where $\overline{u_{i} u_{j}}$ and $\widetilde{u_{i} u_{j}}$ are unknowns, are modeled in terms of eddy viscosity and strain rates at corresponding scales, respectively, or by invoking scale-invariance, as

$$
\tau_{i j}-\frac{\delta_{i j}}{3} \tau_{k k}=-2 C \bar{\Delta}^{2}|\bar{S}| \bar{S}_{i j}=-2 \nu_{t} \bar{S}_{i j}
$$

and

$$
T_{i j}-\frac{\delta_{i j}}{3} T_{k k}=-2 C \tilde{\widetilde{\Delta}}^{2}|\tilde{\bar{S}}| \widetilde{\bar{S}}_{i j}=-2 \nu_{t} \widetilde{\bar{S}}_{i j},
$$


where $\bar{S}_{i j}$ and $\widetilde{\bar{S}}$ refers to the strain rate tensors at the grid- and test- filter levels, respectively. The respective magnitude of strain rates $|\bar{S}|$ and $|\widetilde{\bar{S}}|$ may be written as:

$$
\begin{aligned}
& |\bar{S}|=\sqrt{2 \bar{S}_{i j} \bar{S}_{i j}}, \quad \bar{S}_{i j}=1 / 2\left(\partial_{i} \bar{u}_{j}+\partial_{j} \bar{u}_{i}\right), \\
& |\widetilde{\bar{S}}|=\sqrt{2 \widetilde{\bar{S}}_{i j} \widetilde{\bar{S}}_{i j}}, \quad \widetilde{\bar{S}}_{i j}=1 / 2\left(\partial_{i} \widetilde{\bar{u}}_{j}+\partial_{j} \widetilde{\bar{u}}_{i}\right) .
\end{aligned}
$$

It may be noted that the grid-filtered strain rates can be obtained locally from the non-equilibrium moments, as mentioned above; the test-filtered strain rates can be determined directly from the gradient of test-filtered velocity field, which can, in turn, be obtained by explicit application of test-filter on the grid-filtered velocity field. The expressions for the test-filter that can be conveniently used in the GLBE framework will be discussed later.

The unknown SGS stress at each filter level can be related by the Germano identity [12]

$$
L_{i j}=\widetilde{\bar{u}}_{i} \bar{u}_{j}-\widetilde{\bar{u}_{i}} \widetilde{\bar{u}}_{j}=T_{i j}-\widetilde{\tau}_{i j}
$$

where $L_{i j}$ is the resolved turbulent stress, and upon substituting Eqs. (7) and (8) in Eq. (9), we get an expression between $L_{i j}$ and other known variables with the model coefficient $C$ being the only unknown

$$
L_{i j}-\frac{\delta_{i j}}{3} L_{k k}=-2 C M_{i j}
$$

where

$$
M_{i j}=\widetilde{\bar{\Delta}}^{2}\left|\widetilde{\bar{S}} \widetilde{\bar{S}}_{i j}-\bar{\Delta}^{2}\right| \widetilde{\bar{S} \mid \bar{S}_{i j}}
$$

We note that the computation of the first term in the $M_{i j}$ tensor involves explicit test-filtering and finite-differencing, while its second term can be obtained locally from non-equilibrium moments, as discussed above. Since, the tensor expressions, i.e. Eq. (10) and (11) leads to five independent equations containing $C$, it can be obtained by a least square minimization approach as [14]:

$$
C=-\frac{1}{2} \frac{\left\langle L_{k l} M_{k l}\right\rangle}{\left\langle M_{i j} M_{i j}\right\rangle},
$$

where the usual summation convention of the repeated indices is assumed and $<\cdot>$ represents spatial averaging in homogeneous directions or time averaging 
or both, depending on the problem, so as to stabilize the computations. Also clipping is to be done when $\nu_{t}<0$, i.e. set $\nu_{t}=0$ in such cases. Once the eddy viscosity in Eq. (6) has been determined from Eqs. (9)-(12) it is added to the molecular viscosity $\nu_{0}$, obtained from the statement of the problem, to yield the total viscosity $\nu$, i.e. $\nu=\nu_{0}+\nu_{t}$, which can then be used to compute the "effective" relaxation times for hydrodynamic moments for the GLBE discussed in Sec. 2,

\subsection{Test-Filters}

Several approaches exist to obtain the test-filtered quantities from the gridfiltered variables that can, in turn, be directly obtained from the solution of the GLBE. The approach considered in this paper is the discrete trapezoidal filter [16,68. Discrete trapezoidal filters are employed in this work as they naturally accommodate with the underlying cubic lattice grid structure and thereby allowing an efficient implementation. They involve applying the onedimensional filter successively in each of the three coordinate directions as follows:

$$
\begin{aligned}
& \bar{\phi}_{(i, j, k)}^{*}=1 / 4\left(\bar{\phi}_{(i+1, j, k)}+2 \bar{\phi}_{(i, j, k)}+\bar{\phi}_{(i-1, j, k)}\right), \\
& \bar{\phi}_{(i, j, k)}^{* *}=1 / 4\left(\bar{\phi}_{(i, j+1, k)}^{*}+2 \bar{\phi}_{(i, j, k)}^{*}+\bar{\phi}_{(i, j-1, k)}^{*}\right), \\
& \widetilde{\bar{\phi}}_{(i, j, k)}=1 / 4\left(\bar{\phi}_{(i, j, k+1)}^{* *}+2 \bar{\phi}_{(i, j, k)}^{* *}+\bar{\phi}_{(i, j, k-1)}^{* *}\right),
\end{aligned}
$$

to obtain the test-filtered value $\widetilde{\bar{\phi}}$ from $\bar{\phi}$ at $(i, j, k)$. Near walls, along the coordinate direction $k$, the test-filtered values at the top and bottom can be obtained as $\widetilde{\bar{\phi}}_{(i, j, k)}=1 / 2\left(\bar{\phi}_{(i, j, k)}^{* *}+\bar{\phi}_{(i, j, k+1)}^{* *}\right)$ and $\widetilde{\bar{\phi}}_{(i, j, k)}=1 / 2\left(\bar{\phi}_{(i, j, k-1)}^{* *}+\bar{\phi}_{(i, j, k)}^{* *}\right)$, respectively.

Other types of test-filters specialized for lattice stencils that can also maintain isotropy may also be possible, but presumably at the expense of additional computational overhead. The implementation of test-filters in the multiblock approach (see Sec. 3) leads to additional challenges in how best to deal with performing such filtering operations at the grid boundaries. In order to calculate the model coefficient $C$, it is necessary to use information from two grid spacings away; thus to compute $C$ at the first node on the coarse grid, two ghost nodes are introduced. Velocities at these ghost nodes are obtained by averaging the values at the surrounding fine nodes. Note that since the collision step is not performed on the two fine layers adjacent to the grid boundary, it is not necessary to introduce additional ghost nodes for the fine grid. For an efficient implementation, optimization strategies are considered in the incorporation of the dynamic procedure in the multiblock GLBE. 
It may be noted that the dynamic procedure with other SGS models, such as the dynamic mixed model (DMM) [16] and the dynamic two-parameter model (DTM) [17] can be incorporated in the GLBE in a similar manner. The details of the incorporation of these models in the GLBE are presented in Appendix B.

\section{Results and Discussion}

We will now present investigations of the LBM using the GLBE formulation with dynamic SGS modeling for LES of wall-bounded turbulent flows. In earlier studies, the LBM has been validated as a direct numerical simulations (DNS) approach for such problems [4144]. In the present study, we will assess the performance of GLBE as a LES approach that incorporates SGS effects using a dynamic procedure to compute the model coefficient, thereby avoiding empiricism, on a relatively coarse grid with multiblock approach, while maintaining necessary near-wall resolutions.

\subsection{Turbulent Channel Flow at $R e_{*}=180$}

First, we consider fully-developed turbulent flow in an open channel with a shear Reynolds number of $R e_{*}=u_{*} H / \nu_{0}=180$, where $u_{*}$ is the shear velocity, $\nu_{0}$ is the molecular kinematic viscosity and $H$ is the channel height. The shear

velocity is related to the wall stress $\tau_{w}$ by $u_{*}=\sqrt{\tau_{w} / \rho}$. It represents an important canonical problem to test turbulence models and computational techniques, for which detailed direct numerical simulations (DNS) data are available for comparison [69,70].

\subsubsection{Computational Conditions}

No-slip boundary is considered at the bottom, implemented using half-way link bounce back approach [71, free-slip at the top, by means of specular reflection of distribution functions [24], and periodic boundary conditions are applied in the streamwise, $x$ and spanwise, $y$ directions. The computational domain is chosen with appropriate aspect ratios, viz., $5.7 \mathrm{H}$ and $2.85 \mathrm{H}$ in the streamwise and spanwise directions, respectively. With these aspect ratios, a sufficient number of wall-layer streaks are accommodated and end effects of two-point correlations are excluded, i.e. the two-point velocity correlations in solutions are required to decay nearly to zero within half the domain [72].

For wall-bounded turbulent flows, it is important to adequately resolve the 
near-wall, small-scale turbulent structures. Grid spacing for such problems are generally referred in terms of wall units (designated with a "+" superscript) as $\Delta^{+}=\Delta / \delta_{\nu}$, where $\Delta$ is the wall spacing and $\delta_{\nu}=\nu_{0} / u_{*}$ is the characteristic viscous length scale. When the computations resolve the local dissipative or Kolmogorov length scale $\eta=\left(\nu_{0}^{3} / \epsilon\right)^{1 / 4}$, i.e. $\Delta_{n w}^{+} \leq O\left(\eta^{+}\right)$[72], it can satisfy the near-wall resolution requirements. In particular, it is generally recognized that $1.5 \eta^{+}-2.0 \eta^{+}$represents the upper limit of grid-spacing, above which the small scale turbulent motions in bounded flows are not well resolved. It can be shown by simple arguments that $\eta^{+} \approx 1.5-2.0$ at the wall and that $\eta^{+}$ increases with increasing distance from the wall [2].

To account for resolution requirements, we discretize the computational domain in terms of two blocks with different resolutions, viz., a fine block near the wall and a coarse block, whose grid spacings are twice that of the fineblock in each direction, in the bulk bounded by free-slip surface at the top. For the fine-block, we used $\Delta_{\text {fine }}^{+}=3.95$ in wall units. Due to the use of link-bounce back method for implementation of wall boundary condition, the first lattice node is located at a distance of $\Delta_{n w}^{+}=\Delta^{+} / 2$, which in our case is 1.98. Hence, the computations are expected to fairly resolve the small-scale turbulent structures. For the coarse-block, we used $\Delta_{\text {coarse }}^{+}=2 \times \Delta_{\text {fine }}^{+}=7.90$. The computational domain is discretized by $256 \times 128 \times 12$ grid nodes and $128 \times 64 \times 19$ grid nodes in the fine and coarse blocks, respectively.

The initial mean velocity is specified to satisfy the $1 / 7^{\text {th }}$ power law [2], while initial perturbations satisfying divergence free velocity field [70]. The density field is taken to be $\rho=\rho_{0}=1.0$ for the entire domain. The precise form of the initial fields may not affect the turbulence statistics, but can have significant influence on the convergence of the solution to statistically steady state. In particular, the above choice of initial fields would enable a rapid convergence to the statistically steady state solution of the fluctuating fields obtained by GLBE with forcing term. We employed the consistent initialization procedure for the distribution functions or moments [73, that properly initializes non-equilibrium moments of the GLBE in the presence of non-uniform hydrodynamic fields, such as those mentioned above.

\subsubsection{Computational Procedure}

Using $\vec{F}=-\frac{d p}{d x} \widehat{x}=\frac{\tau_{w}}{H} \widehat{x}=\frac{\rho u_{*}^{2}}{H} \widehat{x}$ as the driving force, LES using the GLBE is performed. The collision step, including the forcing term, is computed in moment space, while the streaming step is carried out in its natural velocity space, and the result of these two steps provides the grid-filtered hydrodynamic fields. The grid-filtered strain rate tensor $\bar{S}_{i j}$ is obtained locally from non-equilibrium moments as shown in Appendix A, which was also found to provide improved numerical stability on coarser grids when used in lieu of 
finite-differencing of the velocity fields. The relaxation process in the collision step is carried out by computing the "effective" relaxation times for hydrodynamic moments obtained from a variable coefficient Smagorinsky SGS eddyviscosity model, whose values are determined by a dynamic procedure. The steps to accomplish this is briefly summarized as follows.

Based on these grid-filtered quantities and considering $\widetilde{\bar{\Delta}} / \bar{\Delta}=2$ where $\bar{\Delta}=\delta_{x}$, discrete trapezoidal test-filters, as discussed in Sec. 4.1, are applied to obtain

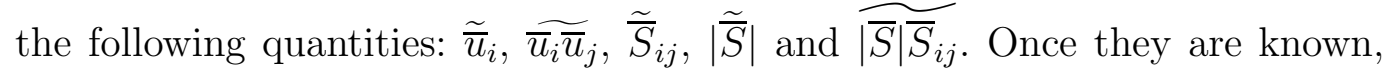
tensors such as $L_{i j}$ and $M_{i j}$ used in the dynamic procedure (Eqs. (9]and[11), respectively, see Sec. 4) are computed. The model coefficient $C$ is, then, obtained by averaging the contracted tensor products, as shown in Eq. (12), along the homogeneous $x$ and $y$ directions, i.e. horizontal planes, to maintain numerical stability. Moreover, near free-slip surfaces, we specify that there is no change in the model coefficient around the top surface to ensure stability. Other SGS models, such as the DTM whose implementation details in GLBE framework are provided in the Appendix $\mathrm{B}$, could be used to relax this assumption, which is a subject for a future work. Finally, the SGS eddy-viscosity is obtained from Eq. (6) to provide the "effective" relaxation times for the collision step. The GLBE computations are carried out until stationary turbulence statistics are obtained, as measured by the invariant Reynolds stresses profiles. This initial run was carried out for a duration of $50 T^{*}$, where $T^{*}=H / u_{*}$ is the characteristic time scale. The averaging of various flow quantities was carried out in time as well as in space in the homogeneous directions by an additional run for a period of $12 T^{*}$.

\subsubsection{Results}

Figure 3 shows the variation of the SGS model coefficient $C_{d y n}=C$ along the wall normal direction, where the distance is scaled in the wall coordinates $Z^{+}$, where $Z^{+}=Z / \delta_{\nu}$ and where $\delta_{\nu}$ is the viscous length scale defined earlier. The model coefficient is seen to be nearly a constant in the bulk or outer flow region, where turbulence tends towards becoming statistically more homogeneous and isotropic. In particular, the value of $C_{d y n}$ in these regions is found to be about 0.024. This is excellent agreement with Germano et al. (1991) [12] based on the solution of filtered Navier-Stokes equations, who found a value of 0.023. The eddy-viscosity given in Eq. (6) , is also written in terms of the Smagorinsky "constant" $C_{S}$ as $\nu_{t}=C_{S}^{2} \bar{\Delta}^{2}|\bar{S}|$. Thus, $C_{S}=\sqrt{C}$ and we find $C_{S}=0.155$ in the bulk region, which is within the range of $0.10-0.16$ reported for various classes of flows [21]. Near $Z^{+}=40$, the grids transition from coarse to fine block, where some oscillations in the values of $C$ are noticed, which could be removed by an additional averaging. Closer to the wall $\left(Z^{+}<30\right)$, in the inner-layer region, the model coefficient is found to decrease monoton- 


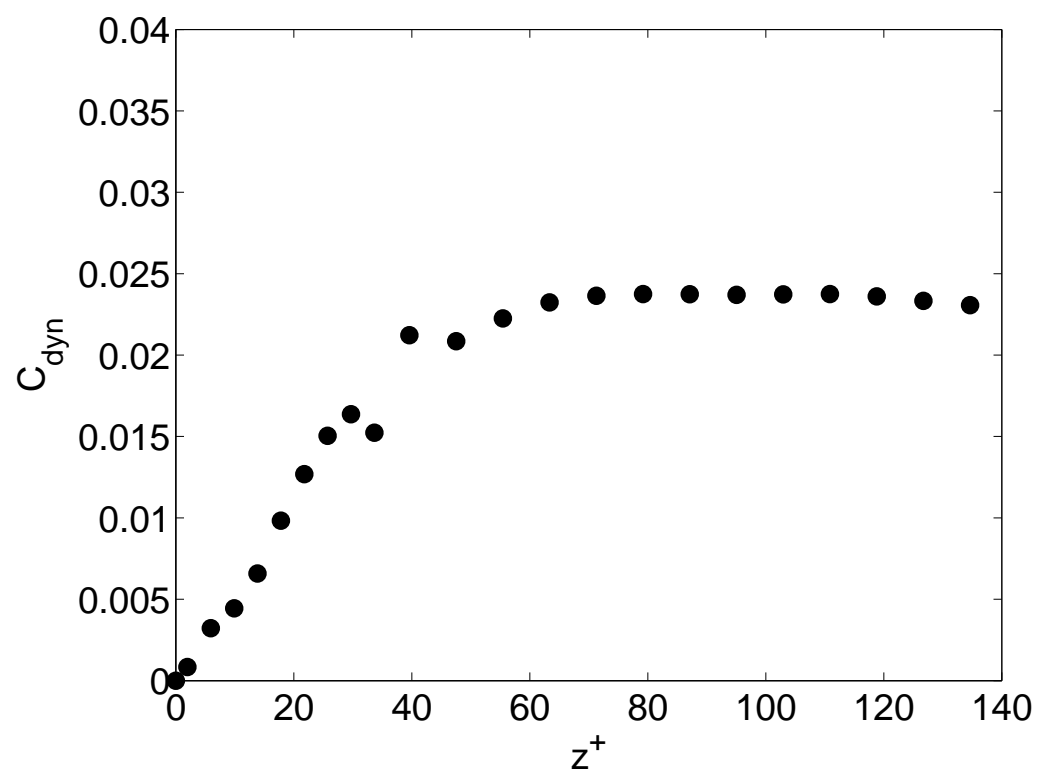

Fig. 3. Computed variation of model coefficient for fully-developed turbulent channel flow with a top slip surface at a shear Reynolds number of $R e_{*}=180$ using a dynamic procedure with multiblock GLBE.

ically with decreasing distance from the wall $Z^{+}$and approaching zero very close to the wall. This trend is consistent with near-wall turbulence physics, according to which turbulence is statistically anisotropic and its scales become smaller towards the wall. Thus, the GLBE using dynamic Smagorinsky SGS model is not only able to self-consistently predict the bulk value of the model coefficient automatically and accurately, but also its variation near the wall without resorting to any empirical approach such as the van Driest damping function [9]. Such predictive capabilities are among the major assets of dynamic SGS modeling using GLBE.

Figure 4 shows the computed mean velocity profile, normalized by the shear velocity $u_{*}$, as a function of the distance from the wall given in wall units, i.e. $Z^{+}$. Also plotted are the DNS data by Kim, Moin and Moser (1987) based on a spectral method 69. The computed velocity profile follows fairly closely with DNS data, with about 5\% difference. Such differences are characteristic of LES, which employ relatively coarser grids than DNS, and which also generally depends on the numerical dissipation of the computational approach for LES (see e.g., Ref. [74,75]).

The Reynolds stress, normalized by the wall-shear stress, is presented in Fig. 5 in semi-log scale and compared with the DNS data of Ref. [69] obtained from the direct solution of incompressible Navier-Stokes equations (NSE). It is found that, in general, the computed Reynolds stress is in reasonably good agreement with DNS. The computed values are somewhat under predicted near $Z^{+}=40$, where the transition between coarse to fine blocks of 


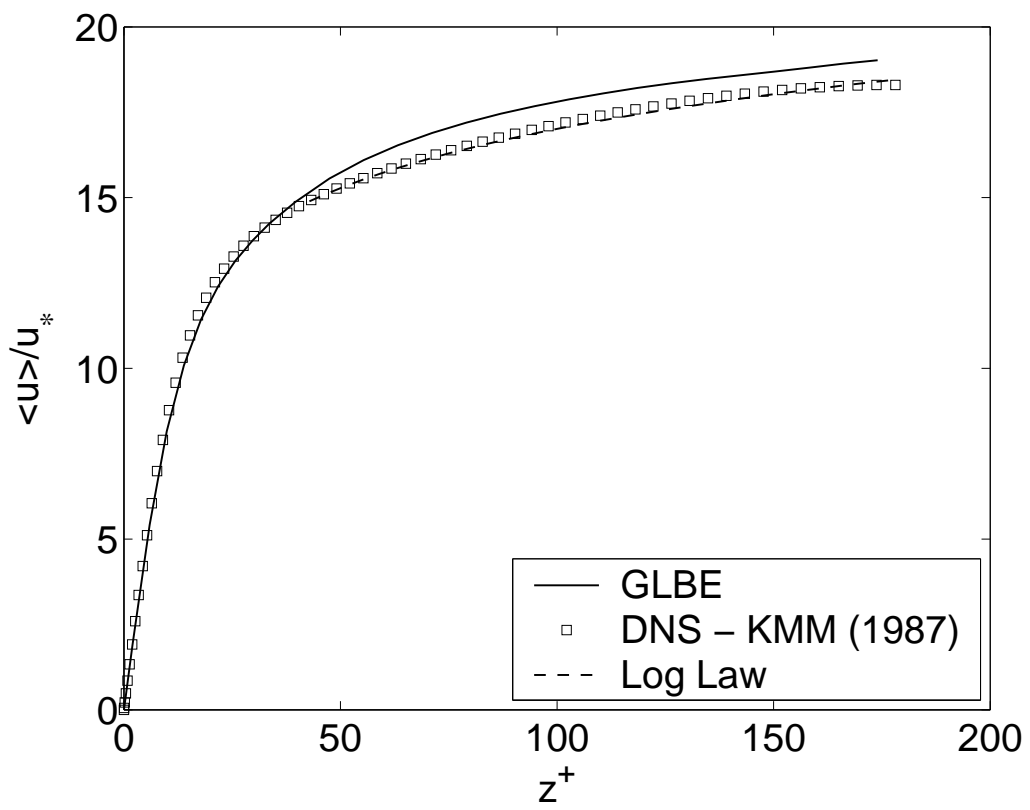

Fig. 4. Mean velocity in fully-developed turbulent channel flow with a top slip surface at a shear Reynolds number of $R e_{*}=180$ using a dynamic procedure with multiblock GLBE. Comparison with DNS of Kim, Moin and Moser (1987).

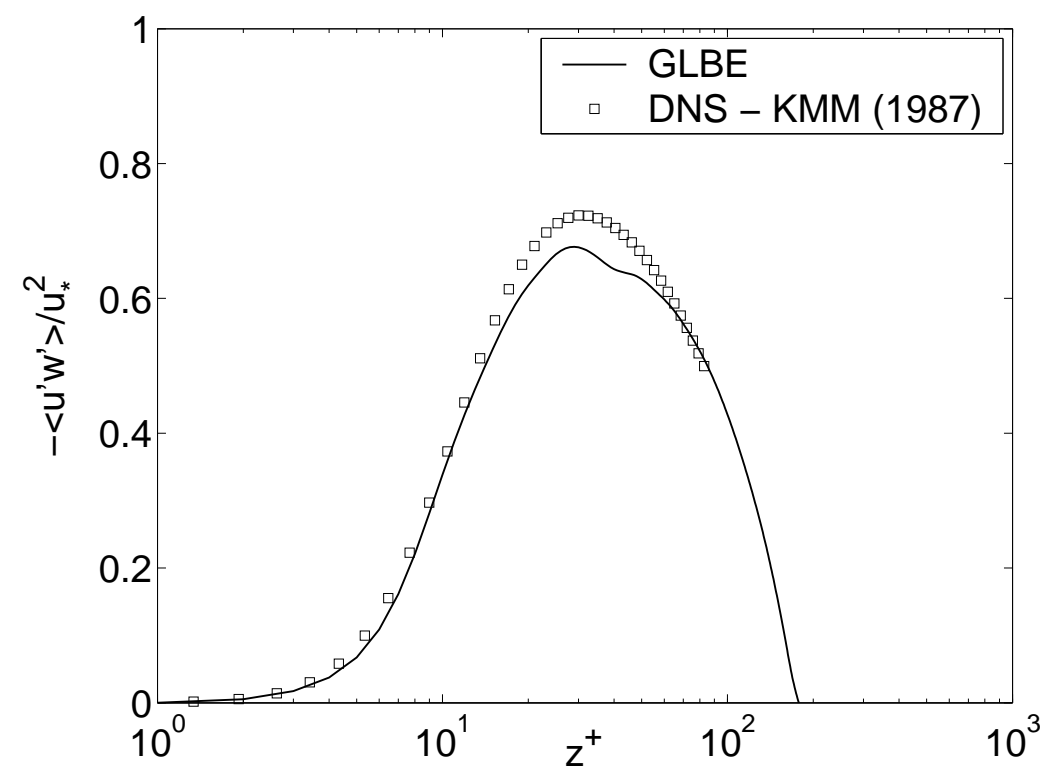

Fig. 5. Mean Reynolds stress in fully-developed turbulent channel flow with a top slip surface at a shear Reynolds number of $R e_{*}=180$ using a dynamic procedure with multiblock GLBE. Comparison with DNS of Kim, Moin and Moser (1987).

grid nodes occur. It is well known that where different types of grids interface, certain amount of numerical effects are inevitable, as was found in certain flow problems 66. Nevertheless, it is clear that the computations are able to predict the Reynolds stress variation both qualitatively and quantitatively in a reasonably accurate manner. 


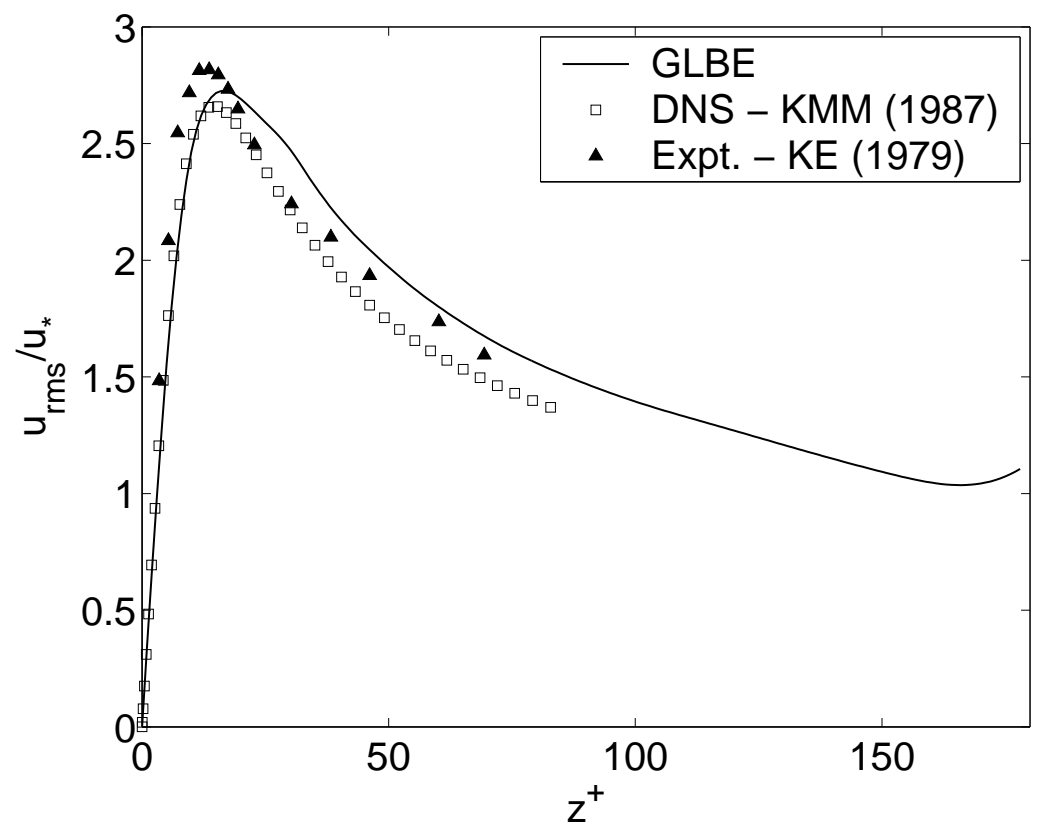

Fig. 6. Root-mean-square(rms) streamwise velocity fluctuations in fully-developed turbulent channel flow with a top slip surface at a shear Reynolds number of $R e_{*}=180$ using a dynamic procedure with multiblock GLBE. Comparison with DNS of Kim, Moin and Moser (1987) and measurements of Kreplin and Eckelmann (1979).

Second-order statistics, such as turbulence intensities are important measures of turbulent activity and let us now assess how the LES computations using the GLBE with a dynamic SGS model are able to predict such quantities in the near-wall region. Figures 6 , 7 and 8 show comparisons of the components of the root-mean-square (rms) streamwise, spanwise and wall-normal velocity fluctuations, respectively, computed using the GLBE with data from DNS based on the NSE [69] and experimental measurements [76]. Again, the computed results are in good agreement with prior data. It is interesting to note among the three components of turbulence intensities, only the wall normal component is the most sensitive to the numerical effects due to coarse-to-fine grid interfaces, which are in any case reasonably small.

\subsection{Turbulent Channel Flow at $R e_{*}=395$}

It is important to know whether the approach discussed in this paper is able to scale well and reproduce turbulence statistics at higher Reynolds numbers. In this regard, we assess the LES approach based on GLBE using dynamic SGS modeling at a higher shear Reynolds number of 395, for which DNS data is available from a more recent work by Moser, Kim and Mansour (1999) [77]. To the best of authors' knowledge, LES using LBM, even with constant Smagorinsky SGS model, at such a higher Reynolds number and 


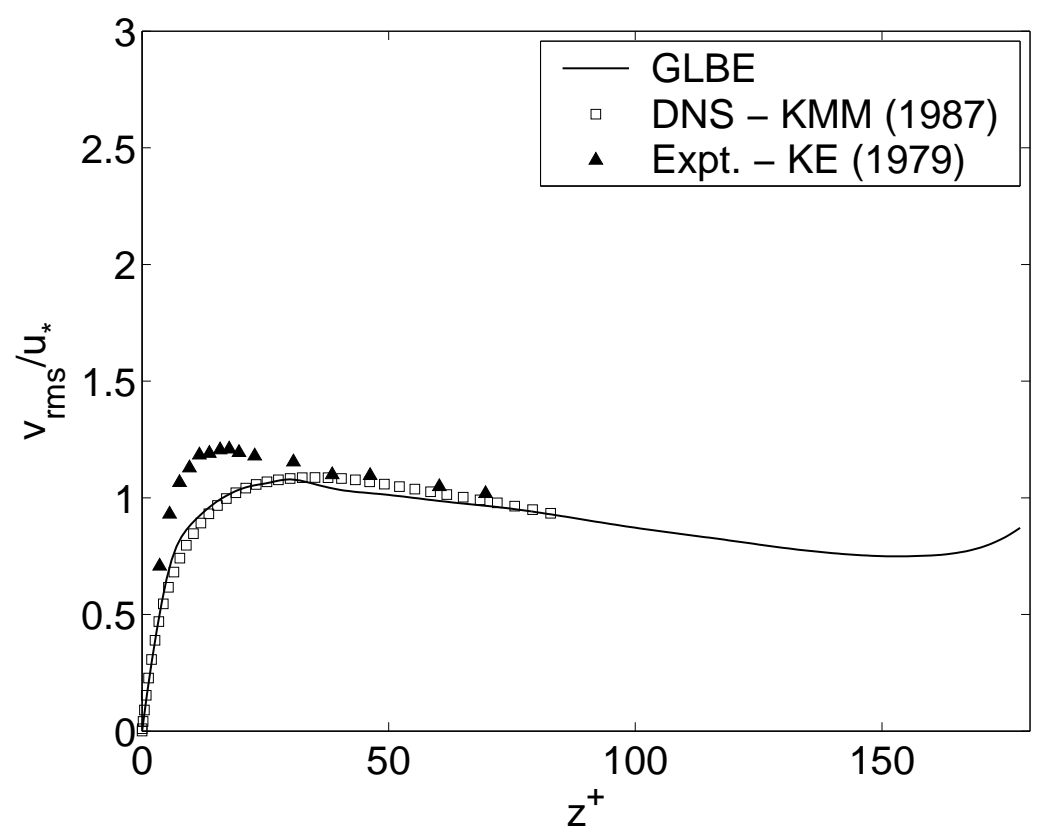

Fig. 7. Root-mean-square(rms) spanwise velocity fluctuations in fully-developed turbulent channel flow with a top slip surface at a shear Reynolds number of $R e_{*}=180$ using a dynamic procedure with multiblock GLBE. Comparison with DNS of Kim, Moin and Moser (1987) and measurements of Kreplin and Eckelmann (1979).

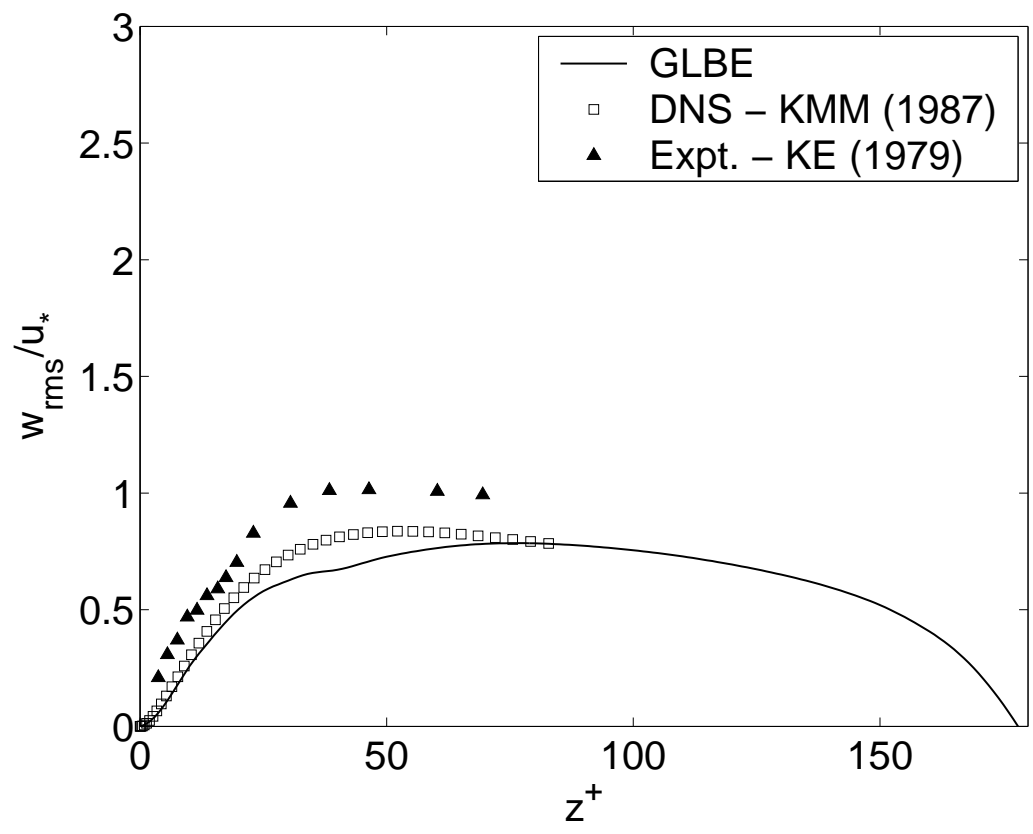

Fig. 8. Root-mean-square(rms) wall normal velocity fluctuations in fully-developed turbulent channel flow with a top slip surface at a shear Reynolds number of $R e_{*}=180$ using a dynamic procedure with multiblock GLBE. Comparison with DNS of Kim, Moin and Moser (1987) and measurements of Kreplin and Eckelmann (1979). 


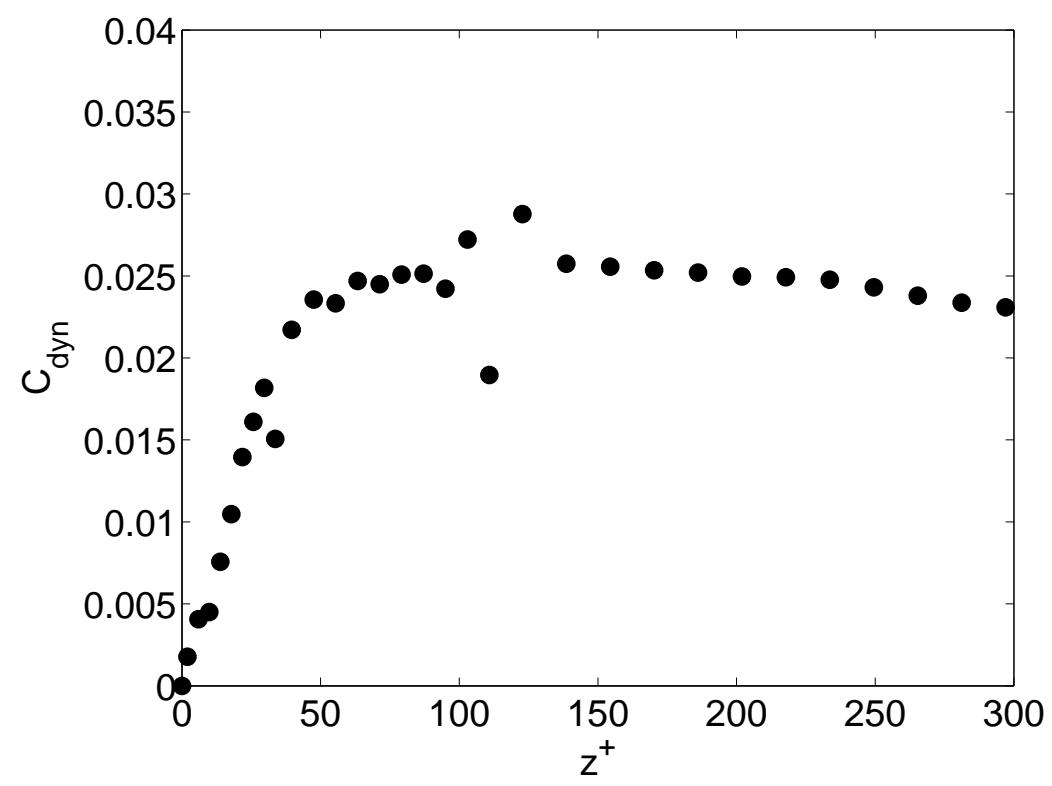

Fig. 9. Computed variation of model coefficient for fully-developed turbulent channel flow with a top slip surface at a shear Reynolds number of $R e_{*}=395$ using a dynamic procedure with multiblock GLBE.

their comparison with the DNS has not previously been reported in the literature. In order to properly sample sufficient number of wall-layer streaks and exclude end effects of two-point velocity correlations, we choose a larger computational domain than in the previous case, with a size of $5.8 H \times 2.9 H \times H$. The computational domain is discretized in terms of four blocks, with the finest grid block near walls, with a spacing of $\Delta^{+}=3.95$. The first grid node is located at $\Delta_{n w}^{+}=\Delta^{+} / 2=1.96$ due to the use of half-way link bounce back, and hence can resolve the smaller near-wall turbulence structures properly. The four blocks are resolved by means of the following number of grid nodes: $576 \times 288 \times 12,288 \times 144 \times 12,144 \times 72 \times 15$ and $288 \times 144 \times 8$, and the GLBE computations are carried out till stationary turbulence are observed, followed by an additional run for a duration of $13 H / u_{*}$ for obtaining turbulence statistics.

Figure 9 shows the computed variation of the model coefficient $C_{d y n}=C$ as a function of the distance from the wall $Z^{+}$for $R e_{*}=395$. Again, the dynamic LES approach is able to predict the value of the model coefficient in the bulk or outer flow region accurately. Moreover, it is also able to reproduce the near-wall trend of $C_{d y n}$ well, viz., its reduction as the distance from the wall is decreased. It is also noticed that around regions, where there is grid block transition, some oscillations in $C_{d y n}$ are present. The magnitude of these oscillations appear to progressively increases with grid transitions involving coarser grid blocks, with the maximum occurring at around $Z^{+}=120$. However, they are generally small and do not seem to affect the turbulent statistics as shown below. 


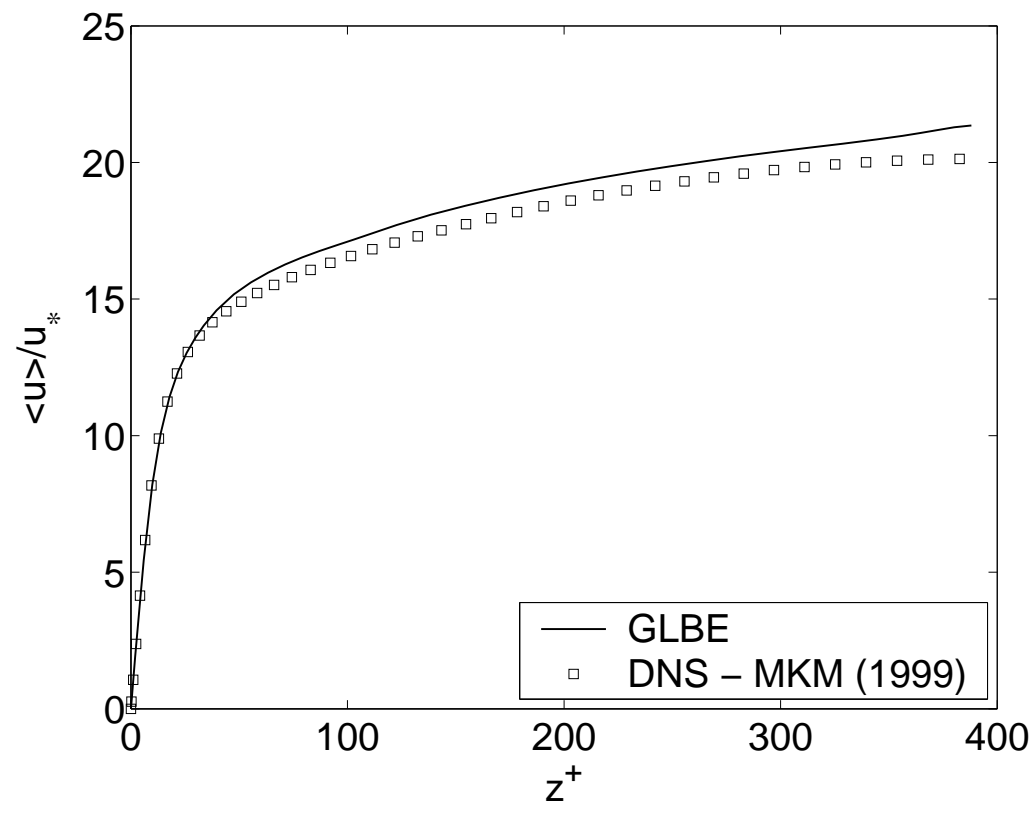

Fig. 10. Mean velocity in fully-developed turbulent channel flow with a top slip surface at a shear Reynolds number of $R e_{*}=180$ using a dynamic procedure with multiblock GLBE. Comparison with DNS of Moser, Kim and Mansour (1999).

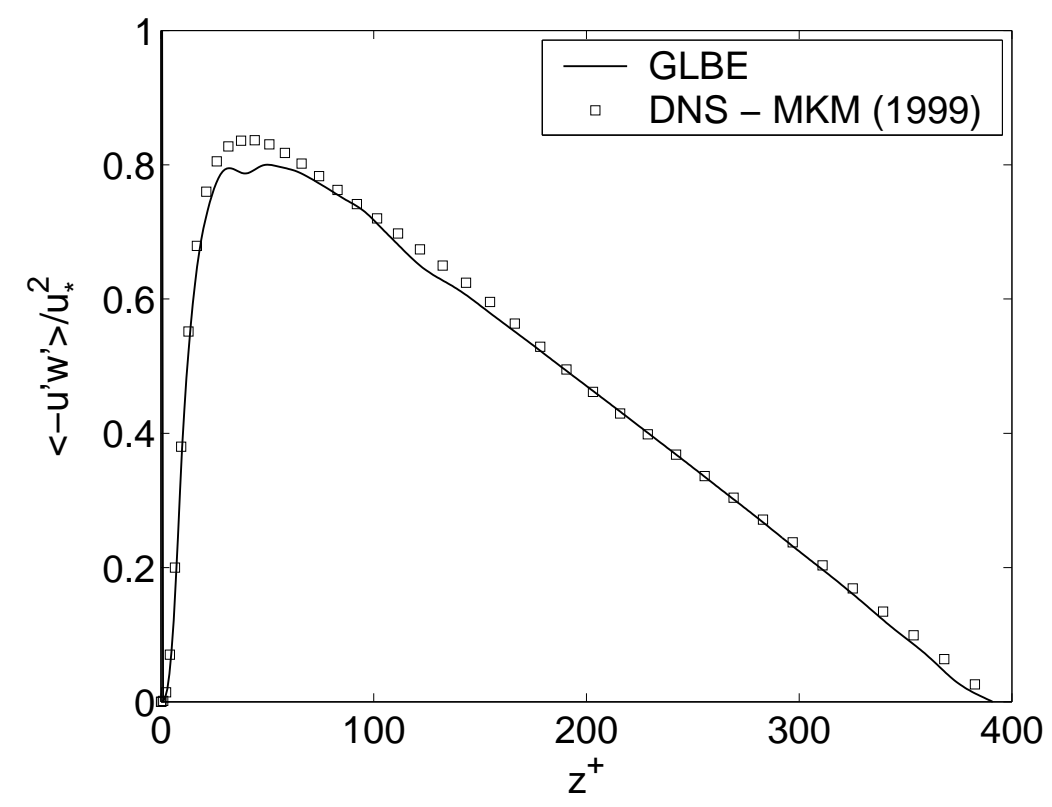

Fig. 11. Mean Reynolds stress in fully-developed turbulent channel flow with a top slip surface at a shear Reynolds number of $R e_{*}=395$ using a dynamic procedure with multiblock GLBE. Comparison with DNS of Moser, Kim and Mansour (1999).

Figure 10 shows the computed mean velocity profile, normalized by the shear velocity $u_{*}$, while the Reynolds stress, normalized by the wall shear stress, for $R e_{*}=395$ is presented in Fig. 11, The DNS data of Moser, Kim and Mansour (1999) [77] are also plotted in these figures for comparison. Notice that the peak Reynolds stress values are higher for the $R e_{*}=395$ case as compared 


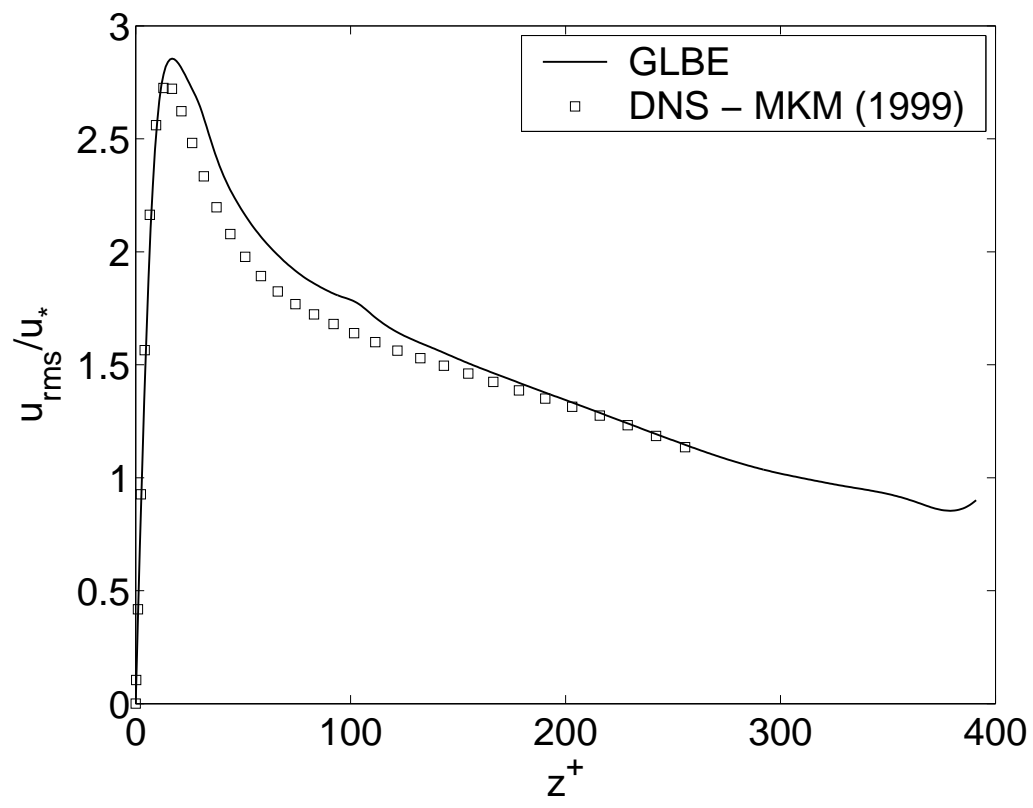

Fig. 12. Root-mean-square(rms) streamwise velocity fluctuations in fully-developed turbulent channel flow with a top slip surface at a shear Reynolds number of $R e_{*}=395$ using a dynamic procedure with multiblock GLBE. Comparison with DNS of Moser, Kim and Mansour (1999).

to the $R e_{*}=180$, reflecting greater turbulent activity at the higher Reynolds number. It is found that the GLBE LES approach using dynamic SGS model is able to reproduce these quantities reasonably well.

Let us now present the root-mean-square (rms) velocity fluctuations in the streamwise, spanwise and wall normal directions in Figs. 12, 13 and 14, respectively. These computed quantities are normalized by the shear velocity and compared with the DNS data [77]. It is noticed that the computed peak values of all the components of turbulent intensities are higher for $R e_{*}=395$ than for $R e_{*}=180$. Moreover, the computed profiles are in good agreement with DNS.

An important indication of how turbulence kinetic energy is transferred among the components is provided by the pressure-strain (PS) correlations. Their components are: $P S_{x}=\left\langle p^{\prime} \partial_{x} u_{x}^{\prime}\right\rangle, P S_{y}=\left\langle p^{\prime} \partial_{y} u_{y}^{\prime}\right\rangle$, and $P S_{z}=\left\langle p^{\prime} \partial_{z} u_{z}^{\prime}\right\rangle$, where the prime denotes fluctuations and the brackets refer to averaging (along homogeneous spatial directions and time). Figure 15 shows the components of the computed PS correlations. They exhibit the expected behavior close to the wall, including the transfer of energy from the wall-normal component to the other two components near the wall - a phenomenon termed as splatting or impingement [10].

It will be interesting to compare the computed results using the dynamic Smagorinsky SGS model with those using the "constant" Smagorinsky SGS 


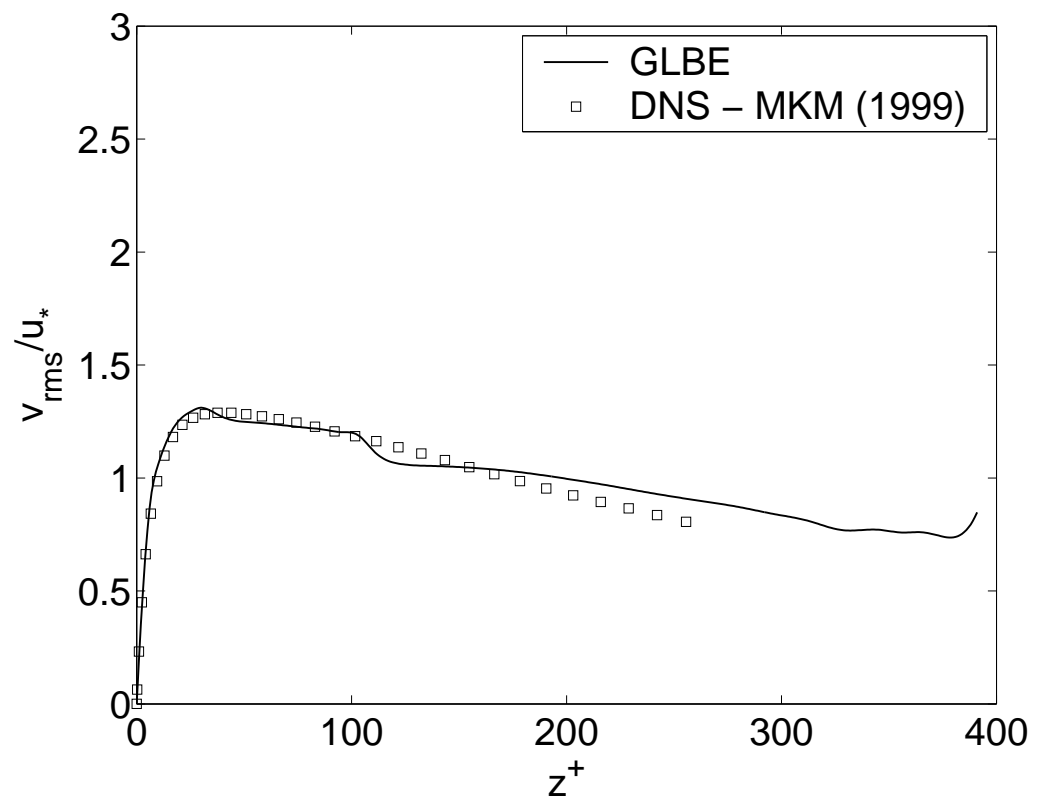

Fig. 13. Root-mean-square(rms) spanwise velocity fluctuations in fully-developed turbulent channel flow with a top slip surface at a shear Reynolds number of $R e_{*}=395$ using a dynamic procedure with multiblock GLBE. Comparison with DNS of Moser, Kim and Mansour (1999).

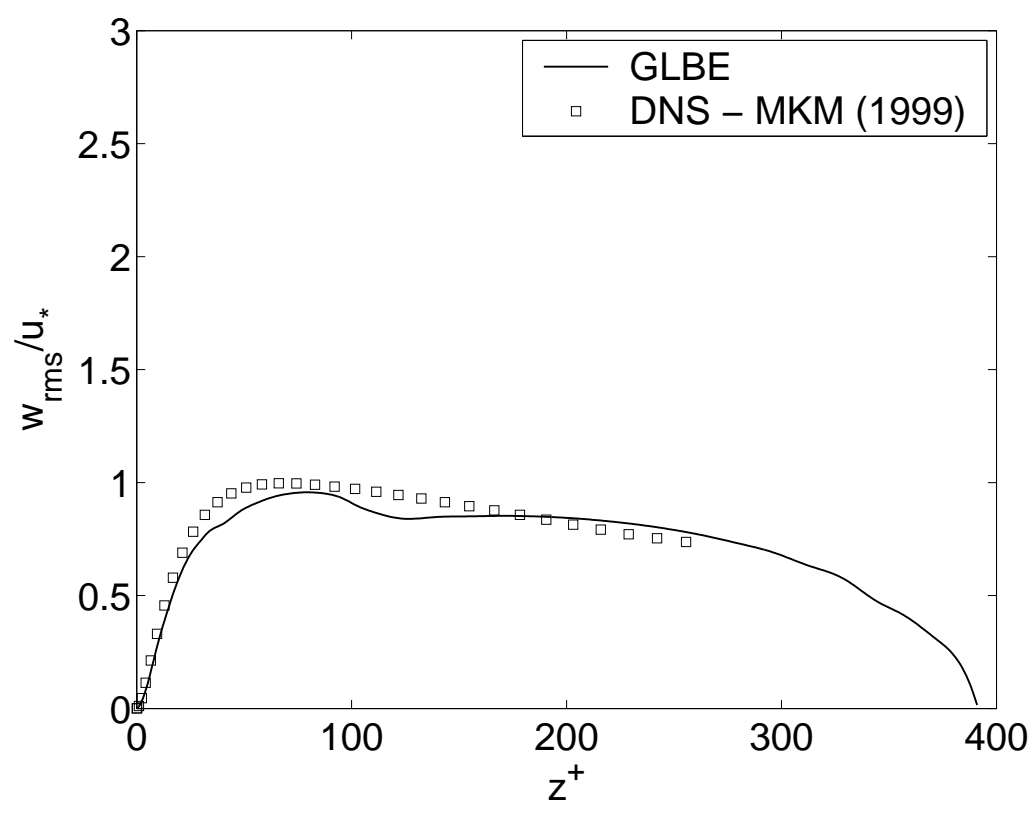

Fig. 14. Root-mean-square(rms) wall normal velocity fluctuations in fully-developed turbulent channel flow with a top slip surface at a shear Reynolds number of $R e_{*}=395$ using a dynamic procedure with multiblock GLBE. Comparison with DNS of Moser, Kim and Mansour (1999). 


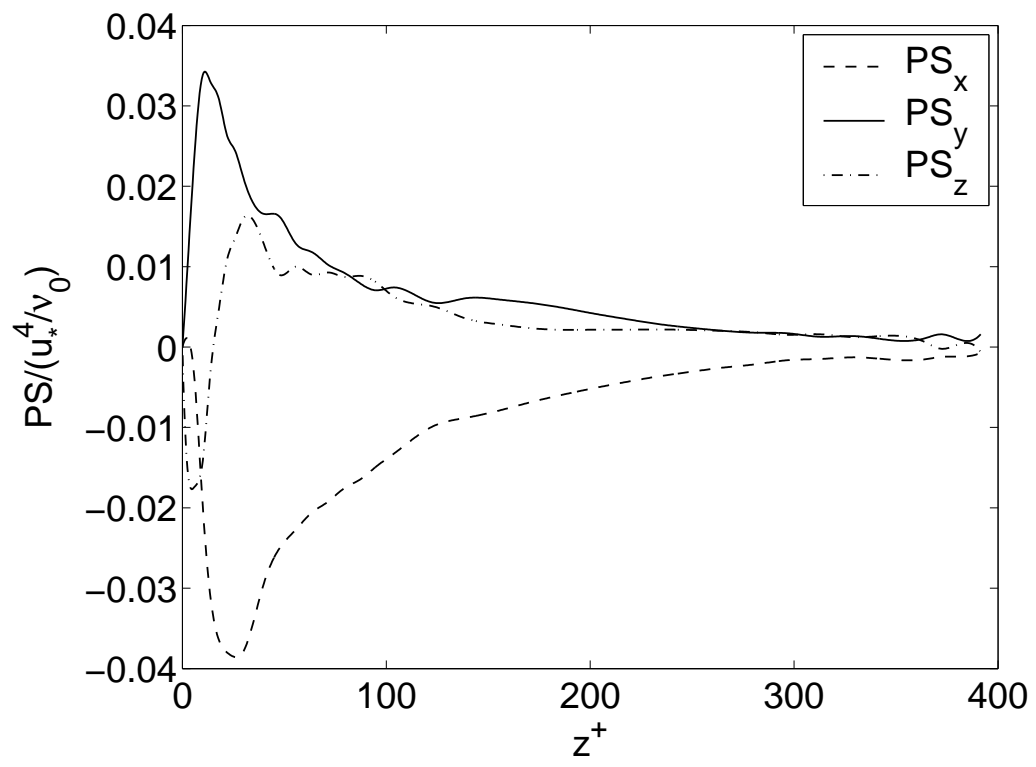

Fig. 15. Components of pressure strain correlations in fully-developed turbulent channel flow with a top slip surface at a shear Reynolds number of $R e_{*}=395$ using a dynamic procedure with multiblock GLBE.

model with an ad hoc van Driest damping function [9]. As an illustration, Fig. [16] shows a comparison of the components of turbulence intensities with both these SGS models. Also, plotted in symbols is the DNS data [77]. In the case of "constant" Smagorinsky SGS model, we consider $C_{S}=0.15$ with the following damping function: $\Delta=\delta_{x}\left[1-\exp \left(-\frac{z^{+}}{A^{+}}\right)\right]$, where $A^{+}=26$. We find that even with the commonly used values of empirical constants, the results with the dynamical SGS model is in better agreement with the DNS. It may be noted that without the use of damping function, the "constant" Smagorinsky SGS model would have given grossly inaccurate results for this problem. In any case, the important point is that the dynamic SGS model, when used with the GLBE for LES, does not require any a priori information regarding the values or the behavior of the model coefficient, which are computed from the local behavior of the turbulent fields itself.

\section{Summary and Conclusions}

In this paper we discussed the incorporation of a dynamic procedure for large eddy simulation (LES) of complex turbulent flows using the generalized lattice Boltzmann equation (GLBE) with forcing term based on multiple relaxation times. The use of dynamic procedure, which samples super-grid or "test" scale turbulent dynamics and assuming scale-invariance between two levels to compute the model coefficient, largely eliminates empiricism required to close the SGS turbulence models, particularly for inhomogeneous and anisotropic 


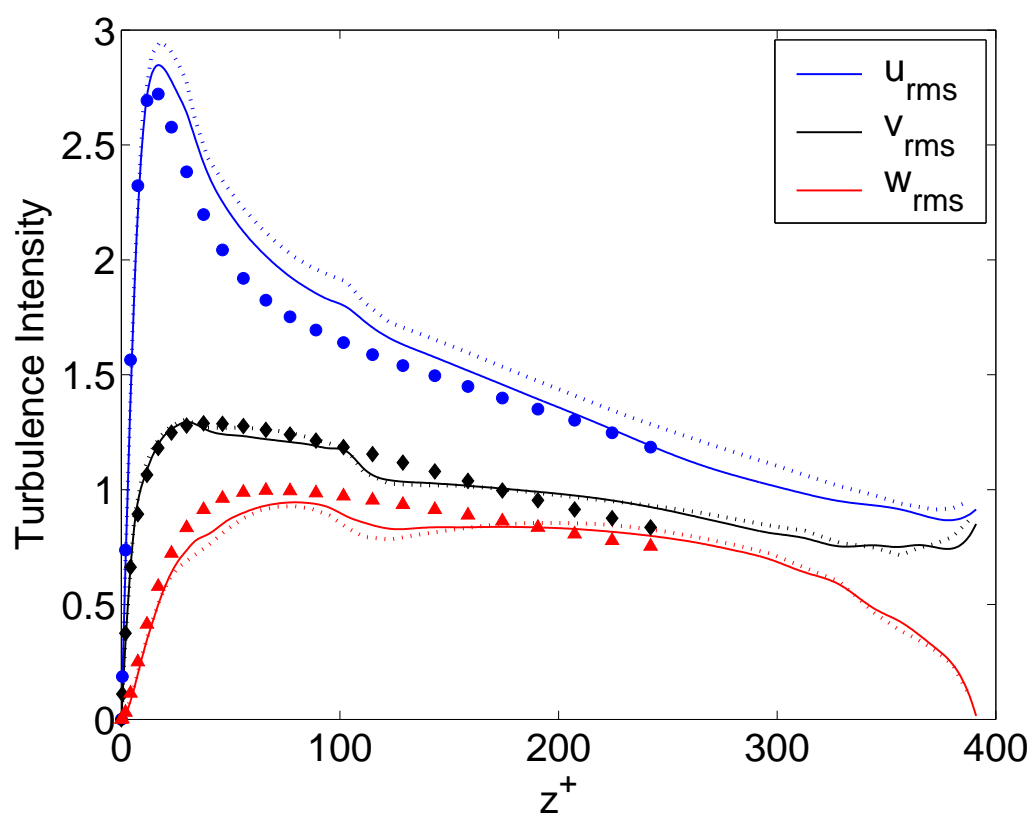

Fig. 16. Comparison between constant (dashed) and dynamic (solid) Smagorinsky models implemented in a multiblock GLBE framework for LES of fully-developed turbulent channel flow with a top slip surface at a shear Reynolds number of $R e_{*}=395$. Symbols are DNS data of Moser, Kim and Mansour (1999). For the constant Smagorinksy model, $C_{s}=0.15$ with van Driest damping using a coefficient of $A^{+}=26$ were considered.

turbulent flows. Eddy-viscosity type dynamic Smagorinsky model (DSM) is employed as the SGS model in this work. The solution of the GLBE, which has significantly improved fidelity and numerical stability when compared to the SRT-LBE due to its ability to separate relaxation time scales of hydrodynamic and kinetic modes, yields the grid-filtered hydrodynamic fields, when "effective" relaxation times based on the dynamic SGS model is employed. Variable resolutions, to accommodate different turbulent length scales with the finest near the walls, are considered by the use of conservative, locally refined multiblock gridding approach. The grid-filtered strain-rate tensor used in the SGS model is computed locally from the non-equilibrium moments of the GLBE, which has superior stability characteristics when compared to the use of simple finite-differencing of the velocity field on coarser grids. To allow efficient implementation, discrete trapezoidal filters are employed to obtain the test-filtered quantities in the dynamic procedure.

Numerical simulation results using the DSM are obtained for the canonical fully-developed turbulent channel flow at shear Reynolds numbers $R e_{*}$ of 180 and 395. It is demonstrated that the use of the multiblock GLBE in conjunction with dynamic procedure for the SGS model is able to automatically compute the values and variation of the model coefficient $C$. In particular, without the use of any ad hoc approach, the computations reproduce the value of $C$ equal to 0.024 (or $C_{S}=0.155$ ) in the bulk flow region, which reduces 
and approaches to zero towards to the wall, reflecting near-wall turbulence physics. The computed turbulent statistics such as the root-mean-square velocity fluctuations and Reynolds stresses for both cases of Reynolds numbers are in good agreement with prior direct numerical simulations (DNS) and experimental data, as applicable. In particular, the peaks of the second-order statistics scale to higher values at higher $R e_{*}$, which the computations are found to reproduce quantitatively well. While the focus of this paper, as a first step, is on the use of dynamic procedure for eddy-viscosity based models in the LBM framework, it also outlines the details of implementation of more general scale-similarity based dynamic SGS models. It appears that the use of dynamic procedure in the LBM, particularly with the GLBE, is a promising approach for LES of complex turbulent flows.

\section{Acknowledgements}

This work was performed under the auspices of the National Aeronautics and Space Administration (NASA) under Contract No. NNL07AA04C. Computational resources were provided by the National Center for Supercomputing

Applications (NCSA) under Award CTS 060027 and the Office of Science of DOE under Contract DE-AC03-76SF00098. 


\section{A Moments, Equilibrium Moments, Moment-projections of Source Terms for D3Q19 Model}

The components of the various elements in the moments are as follows [56]:

$\widehat{f}_{0}=\rho, \widehat{f}_{1}=e, \widehat{f}_{2}=e^{2}, \widehat{f}_{3}=j_{x}, \widehat{f}_{4}=q_{x}, \widehat{f}_{5}=j_{y}, \widehat{f}_{6}=q_{y}, \widehat{f}_{7}=j_{z}, \widehat{f}_{8}=q_{z}, \widehat{f}_{9}=$ $3 p_{x x}, \widehat{f}_{10}=3 \pi_{x x}, \widehat{f}_{11}=p_{w w}, \widehat{f}_{12}=\pi_{w w}, \widehat{f}_{13}=p_{x y}, \widehat{f}_{14}=p_{y z}, \widehat{f}_{15}=p_{x z}, \widehat{f}_{16}=$ $m_{x}, \widehat{f}_{17}=m_{y}, \widehat{f}_{18}=m_{z}$. Here, $\rho$ is the density, $e$ and $e^{2}$ represent kinetic energy that is independent of density and square of energy, respectively; $j_{x}$, $j_{y}$ and $j_{z}$ are the components of the momentum, i.e. $j_{x}=\rho u_{x}, j_{y}=\rho u_{y}$, $j_{z}=\rho u_{z}, q_{x}, q_{y}, q_{z}$ are the components of the energy flux, and $p_{x x}, p_{x y}, p_{y z}$ and $p_{x z}$ are the components of the symmetric traceless viscous stress tensor; The other two normal components of the viscous stress tensor, $p_{y y}$ and $p_{z z}$, can be constructed from $p_{x x}$ and $p_{w w}$, where $p_{w w}=p_{y y}-p_{z z}$. Other moments include $\pi_{x x}, \pi_{w w}, m_{x}, m_{y}$ and $m_{z}$. The first two of these moments have the same symmetry as the diagonal part of the traceless viscous tensor $p_{i j}$, while the last three vectors are parts of a third rank tensor, with the symmetry of $j_{k} p_{m n}$.

The corresponding components of the equilibrium moments, which are functions of conserved moments, i.e. density $\rho$ and momentum $\vec{j}$, are as follows [56]:

$\widehat{f}_{0}^{e q}=\rho, \widehat{f}_{1}^{e q} \equiv e^{e q}=-11 \rho+19 \frac{\vec{j} \cdot \vec{j}}{\rho}, \widehat{f}_{2}^{e q} \equiv e^{2, e q}=3 \rho-\frac{11}{2} \frac{\vec{j} \cdot \vec{j}}{\rho}, \widehat{f}_{3}^{e q}=j_{x}, \widehat{f}_{4}^{e q} \equiv$ $q_{x}^{e q}=-\frac{2}{3} j_{x}, \widehat{f}_{5}^{e q}=j_{y}, \widehat{f}_{6}^{e q} \equiv q_{y}^{e q}=-\frac{2}{3} j_{y}, \hat{f}_{7}^{e q}=j_{z}, \widehat{f}_{8}^{e q} \equiv q_{z}^{e q}=-\frac{2}{3} j_{z}, \widehat{f}_{9}^{e q} \equiv$ $3 p_{x x}^{e q}=\frac{\left[3 j_{x}^{2}-\vec{j} \cdot \vec{j}\right]}{\rho}, \widehat{f}_{10}^{e q} \equiv 3 \pi_{x x}^{e q}=3\left(-\frac{1}{2} p_{x x}^{e q}\right), \widehat{f}_{11}^{e q} \equiv p_{w w}^{e q}=\frac{\left[j_{y}^{2}-j_{z}^{2}\right]}{\rho}, \widehat{f}_{12}^{e q} \equiv$ $\pi_{w w}^{e q}=-\frac{1}{2} p_{w w}^{e q}, \widehat{f}_{13}^{e q} \equiv p_{x y}^{e q}=\frac{j_{x} j_{y}}{\rho}, \widehat{f}_{14}^{e q} \equiv p_{y z}^{e q}=\frac{j_{y} j_{z}}{\rho}, \widehat{f}_{15}^{e q} \equiv p_{x z}^{e q}=\frac{j_{x} j_{z}}{\rho}, \widehat{f}_{16}^{e q}=$ $0, \hat{f}_{17}^{e q}=0, \hat{f}_{18}^{e q}=0$.

The components of the source terms in moment space are functions of external force $\vec{F}$ and velocity fields $\vec{u}$, respectively, as follows [62]:

$\widehat{S}_{0}=0, \widehat{S}_{1}=38\left(F_{x} u_{x}+F_{y} u_{y}+F_{z} u_{z}\right), \widehat{S}_{2}=-11\left(F_{x} u_{x}+F_{y} u_{y}+F_{z} u_{z}\right), \widehat{S}_{3}=$ $F_{x}, \widehat{S}_{4}=-\frac{2}{3} F_{x}, \widehat{S}_{5}=F_{y}, \widehat{S}_{6}=-\frac{2}{3} F_{y}, \widehat{S}_{7}=F_{z}, \widehat{S}_{8}=-\frac{2}{3} F_{z}, \widehat{S}_{9}=2\left(2 F_{x} u_{x}-\right.$ $\left.F_{y} u_{y}-F_{z} u_{z}\right), \widehat{S}_{10}=-\left(2 F_{x} u_{x}-F_{y} u_{y}-F_{z} u_{z}\right), \widehat{S}_{11}=2\left(F_{y} u_{y}-F_{z} u_{z}\right), \widehat{S}_{12}=$ $-\left(F_{y} u_{y}-F_{z} u_{z}\right), \widehat{S}_{13}=\left(F_{x} u_{y}+F_{y} u_{x}\right), \widehat{S}_{14}=\left(F_{y} u_{z}+F_{z} u_{y}\right), \widehat{S}_{15}=\left(F_{x} u_{z}+\right.$ $\left.F_{z} u_{x}\right), \widehat{S}_{16}=0, \widehat{S}_{17}=0, \widehat{S}_{18}=0$.

The components of the strain rate tensor used in subgrid scale (SGS) turbulence model at the grid-filter level can be written explicitly in terms of non-equilibrium moments augmented by moment-projections of source terms as 62 ] 


$$
\begin{aligned}
& S_{x x} \approx-\frac{1}{38 \rho}\left[s_{1} \widehat{h}_{1}^{(n e q)}+19 s_{9} \widehat{h}_{9}^{(n e q)}\right], \\
& S_{y y} \approx-\frac{1}{76 \rho}\left[2 s_{1} \widehat{h}_{1}^{(n e q)}-19\left(s_{9} \widehat{h}_{9}^{(n e q)}-3 s_{11} \widehat{h}_{11}^{(n e q)}\right)\right], \\
& S_{z z} \approx-\frac{1}{76 \rho}\left[2 s_{1} \widehat{h}_{1}^{(n e q)}-19\left(s_{9} \widehat{h}_{9}^{(n e q)}+3 s_{11} \widehat{h}_{11}^{(n e q)}\right)\right], \\
& S_{x y} \approx-\frac{3}{2 \rho} s_{13} \widehat{h}_{13}^{(n e q)} \\
& S_{y z} \approx-\frac{3}{2 \rho} s_{14} \widehat{h}_{14}^{(n e q)} \\
& S_{x z} \approx-\frac{3}{2 \rho} s_{15} \widehat{h}_{15}^{(n e q)}
\end{aligned}
$$

where

$$
\widehat{h}_{\alpha}^{(n e q)}=\widehat{f}_{\alpha}-\widehat{f}_{\alpha}^{e q}+\frac{1}{2} \widehat{S}_{\alpha}, \quad \alpha \in\{1,9,11,13,14,15\}
$$

Here, $\widehat{f}_{\alpha}, \widehat{f}_{\alpha}^{e q}$ and $\widehat{S}_{\alpha}$ are components of moments, their local equilibria, and moment-projections of source terms due to external forces, respectively, which are given above. $s_{\alpha}$ are elements of the collision matrix $\widehat{\Lambda}=\operatorname{diag}\left(s_{0}, s_{1}, \ldots, s_{18}\right)$ in moment space. The expressions for the strain rate tensor are generalizations of those given in $\mathrm{Yu}$ et al. [50]

\section{B Incorporation of Dynamic Mixed Model (DMM) and Dynamic Two Parameter (DTM) Subgrid Scale Models in Lattice-Boltzmann Method}

It is well recognized that improvements to the dynamic Smagorinsky model (DSM) is required to properly account for various aspects of subgrid scale turbulence physics in general situations. For example, while DSM is generally adequate for performing large-eddy simulation (LES) of bulk as well as nearwall turbulent flows, it is less satisfactory near the free-slip or free-surfaces. This is presumably because of the assumption in the DSM that turbulence dissipation is in equilibrium with production, leading to the eddy-viscosity type models, which implies the alignment of the principal axes of the SGS stress tensor with those of the resolved strain rate tensor. Moreover, when the dynamic coefficient $C$ is computed locally, it exhibits large fluctuations leading to excessive energy backscatter and numerical instability. Thus, the DSM can accurately predict mean flow quantities when an averaged value of the coefficient is employed, but is inadequate for representation of the local quantities. 
Thus, Zang et al. (1993) [16] proposed to circumvent these problems by employing a mixed model, which also contains contributions from the scalesimilarity based stresses or the modified Leonard stress terms (as originally proposed by Bardina et al. (1980) [15]) and do not assume the alignment of the SGS stress and the resolved strain rate tensors. The proportionality constant for the Leonard stress terms is set to 1.0 in this model. By alleviating the burden on the variation of the coefficient $C$ to represent turbulence physics, this dynamic mixed model (DMM) provided improved results, with significantly less fluctuations in the model coefficient. A further improvement, particularly in the context of LES of free-surface turbulence, was provided by the dynamic two-parameter model (DTM) of Salvetti and Banerjee (1995) [17]. In this model, an additional parameter $K$ is introduced as a proportionality constant to the modified Leonard stress term in the mixed based model. Both the coefficients $C$ and $K$ can then be obtained locally by means of a least-squares technique, as formulated by Lilly (1992) [14]. We now discuss the development of a procedure for incorporation of DTM in the framework of lattice Boltzmann method (LBM).

The anisotropic part of the SGS stress in the DTM can be represented as

$$
\tau_{i j}-\frac{\delta_{i j}}{3} \tau_{k k}=-2 C \bar{\Delta}^{2}|\bar{S}| \bar{S}_{i j}+K\left[L_{i j}^{m}-\frac{\delta_{i j}}{3} L_{k k}^{m}\right],
$$

where the first term on the right-hand-side (RHS) is due to the eddy-viscosity Smagorinsky model, while the second term is the modified Leonard stress term $L_{i j}^{m}$, which is given by

$$
L_{i j}^{m}=\overline{\bar{u}}_{i} \bar{u}_{j}-\overline{\bar{u}}_{i} \overline{\bar{u}}_{j}
$$

The modified Leonard stress is a known quantity from the resolved field. At the test filter level, the SGS stress can be written as

$$
T_{i j}-\frac{\delta_{i j}}{3} T_{k k}=-2 C \tilde{\widetilde{\Delta}}^{2}|\widetilde{\bar{S}}| \widetilde{\bar{S}}_{i j}+K\left[L_{i j}^{t}-\frac{\delta_{i j}}{3} L_{k k}^{t}\right] .
$$

Using Germano's identity (1991) [12], we obtain

$$
L_{i j}=T_{i j}-\widetilde{\tau}_{i j}
$$

which leads to

$$
L_{i j}-\frac{\delta_{i j}}{3} L_{k k}=-2 C \bar{\Delta}^{2} M_{i j}+K\left[H_{i j}-\frac{\delta_{i j}}{3} H_{k k}\right],
$$


where

$$
\begin{aligned}
L_{i j} & ={\widetilde{u_{i} \bar{u}_{j}}}-\widetilde{\bar{u}}_{i} \widetilde{\bar{u}}_{j}, \\
H_{i j} & =\overline{\overline{\bar{u}}}_{i} \overline{\bar{u}}_{j}-\widetilde{\bar{u}}_{i} \widetilde{\overline{\bar{u}}}_{j}, \\
M_{i j} & =\alpha^{2} \mid \widetilde{\bar{S}}^{\widetilde{S}_{i j}}-\widetilde{\bar{S} \mid \bar{S}_{i j}} .
\end{aligned}
$$

Here, all the above tensors, $L_{i j}, H_{i j}$ and $M_{i j}$, are known from the resolved field and $\alpha=\tilde{\bar{\Delta}} / \bar{\Delta}$ is the ratio of test-filter and grid-filter sizes. To obtain the constants $C$ and $K$, the least squares technique in the context of dynamic SGS modeling as proposed by Lilly (1992) [14] is employed. Thus, the constants are obtained by minimizing the following function with respect to these parameters:

$$
Q=\left\{L_{i j}-\frac{\delta_{i j}}{3} L_{k k}+2 C \bar{\Delta}^{2} M_{i j}-K\left[H_{i j}-\frac{\delta_{i j}}{3} H_{k k}\right]\right\}^{2} .
$$

With $\partial Q / \partial C=0$ and $\partial Q / \partial K=0$, we obtain

$$
\begin{aligned}
& K=\frac{a b / d-m}{a^{2} / d-n}, \\
& C=\frac{a K-b}{2 \bar{\Delta}^{2} d}
\end{aligned}
$$

where $a=\left[H_{i j}-\frac{\delta_{i j}}{3} H_{k k}\right] M_{i j}, b=\left[L_{i j}-\frac{\delta_{i j}}{3} L_{k k}\right] M_{i j}, d=M_{i j} M_{i j}, m=$ $\left[L_{i j}-\frac{\delta_{i j}}{3} L_{k k}\right]\left[H_{i j}-\frac{\delta_{i j}}{3} H_{k k}\right]$, and $n=\left[H_{i j}-\frac{\delta_{i j}}{3} H_{k k}\right]\left[H_{i j}-\frac{\delta_{i j}}{3} H_{k k}\right]$.

It may be noted that when DMM is employed, with $K=1$, the Smagorinsky coefficient becomes

$$
C=\frac{a K-b}{2 \bar{\Delta}^{2} d}=\frac{\left[\left(L_{i j}-\frac{\delta_{i j}}{3} L_{k k}\right)-\left(H_{i j}-\frac{\delta_{i j}}{3} H_{k k}\right)\right]}{2 \bar{\Delta}^{2} M_{i j} M_{i j}} .
$$

Once the local values of the coefficients $C$ and $K$ are known, the SGS stress can be incorporated within the framework of lattice Boltzmann method (LBM) as follows: The first part of the SGS stress in DTM provides the eddy viscosity

$$
\nu_{t}=C \bar{\Delta}^{2}|\bar{S}|
$$

which can be added to the molecular viscosity $\nu_{0}$, obtained from the statement of the problem, to yield the total viscosity $\nu$, i.e., $\nu=\nu_{0}+\nu_{t}$ can then be 
used to compute relaxation times $\left(s_{10}, s_{12}, s_{14}, s_{15}, s_{16}\right)$ in the MRT-LBE, as discussed in the main text of this paper for the DSM. The second part of the SGS stress can be introduced as forcing terms in the MRT-LBE. Thus, the modified Leonard stress can be written as the following Cartesian components of the effective force:

$$
\begin{aligned}
& F_{x}^{m L}=-\left[\partial_{x}\left(K L_{x x}^{m}\right)+\partial_{y}\left(K L_{x y}^{m}\right)+\partial_{z}\left(K L_{x z}^{m}\right)\right] \\
& F_{y}^{m L}=-\left[\partial_{x}\left(K L_{y x}^{m}\right)+\partial_{y}\left(K L_{y y}^{m}\right)+\partial_{z}\left(K L_{y z}^{m}\right)\right] \\
& F_{z}^{m L}=-\left[\partial_{x}\left(K L_{z x}^{m}\right)+\partial_{y}\left(K L_{z y}^{m}\right)+\partial_{z}\left(K L_{z z}^{m}\right)\right] .
\end{aligned}
$$

The spatial derivatives needed in the forcing terms can be evaluated by employing isotropic spatial discrete derivative operators based on lattice stencils. Such forcing terms $\vec{F}^{m L}$, where they are added to $\vec{F}$ enter the MRT-LBE through the source terms in the moment space $\widehat{S}_{\alpha}$, defined at the end of Appendix A.

It may be noted that the grid-filtered fields can be obtained directly from the solution of the MRT-LBE. On the other hand, the double-grid filtered $\overline{\bar{\phi}}$ as well as the test-filtered value $\widehat{\bar{\phi}}$, required in the DMM as well as the DSM, can be obtained from the repeated application of trapezoidal discrete filters for the grid and test volumes, respectively, successively for each spatial dimension [16]. Thus, at grid node $(i, j, k)$, for the double-grid filter:

$$
\begin{aligned}
& \bar{\phi}_{(i, j, k)}^{*}=1 / 8\left(\bar{\phi}_{(i+1, j, k)}+6 \bar{\phi}_{(i, j, k)}+\bar{\phi}_{(i-1, j, k)}\right), \\
& \bar{\phi}_{(i, j, k)}^{* *}=1 / 8\left(\bar{\phi}_{(i, j+1, k)}^{*}+6 \bar{\phi}_{(i, j, k)}^{*}+\bar{\phi}_{(i, j-1, k)}^{*}\right), \\
& \overline{\bar{\phi}}_{(i, j, k)}=1 / 8\left(\bar{\phi}_{(i, j, k+1)}^{* *}+6 \bar{\phi}_{(i, j, k)}^{* *}+\bar{\phi}_{(i, j, k-1)}^{* *}\right),
\end{aligned}
$$

and for the test-filter:

$$
\begin{aligned}
& \bar{\phi}_{(i, j, k)}^{*}=1 / 4\left(\bar{\phi}_{(i+1, j, k)}+2 \bar{\phi}_{(i, j, k)}+\bar{\phi}_{(i-1, j, k)}\right), \\
& \bar{\phi}_{(i, j, k)}^{* *}=1 / 4\left(\bar{\phi}_{(i, j+1, k)}^{*}+2 \bar{\phi}_{(i, j, k)}^{*}+\bar{\phi}_{(i, j-1, k)}^{*}\right), \\
& \widetilde{\bar{\phi}}_{(i, j, k)}=1 / 4\left(\bar{\phi}_{(i, j, k+1)}^{* *}+2 \bar{\phi}_{(i, j, k)}^{* *}+\bar{\phi}_{(i, j, k-1)}^{* *}\right) .
\end{aligned}
$$

It may be noted that implementation of this approach in the MRT-LBE within the context of multiblock grids would require careful consideration of information exchange between different grid levels in the grid transition regions. Implementation and assessment of dynamic scale-similarity based SGS models in the GLBE framework are subjects of future investigations. 


\section{References}

[1] U. Frisch, Turbulence: The Legacy of A.N. Kolmogorov, Cambridge University Press, New York (1995).

[2] S. Pope, Turbulent Flows, Cambridge University Press, New York (2000).

[3] M. Lesieur, Turbulence in Fluids, Springer, New York (2007).

[4] J. Smagorinsky, General Circulation Experiments with the Primitive Equations, Mon. Wea. Rev. 91 (1963) 99.

[5] D. Lilly, On the Application of the Eddy Viscosity Concept in the Inertial Subrange of Turbulence, NCAR Manuscript 123 National Center for Atmospheric Research, Boulder, CO (1966).

[6] J. Deardorff, A Numerical Study of Three-dimensional Channel Flow at Large Reynolds Numbers, J. Fluid Mech. 41 (1970) 453.

[7] U. Schumann, Subgrid Scale Model for Finite Difference Simulations of Turbulent Flows in Plane Channels and Annuli, J. Comp. Phys. 18 (1975) 376.

[8] A. Leonard, Energy Cascade in Large-Eddy Simulations of Turbulent Fluids Flows, Adv. Geo. Phys. 18A (1974) 237.

[9] E. van Driest, On Turbulent Flow Near a Wall, J. Aeronaut Sci. 23 (1956) 1007.

[10] P. Moin and J. Kim, Numerical Investigation of Turbulent Channel Flow, J. Fluid Mech. 118 (1982) 341.

[11] U. Piomelli, T. Zang, C. Speziale and M.Y. Hussaini, On the Large-Eddy Simulation of Wall-Bounded Transitional Flows, Phys. Fluids A 2 (1990) 257.

[12] M. Germano, U. Piomelli, P. Moin and W. Cabot, A Dynamic Subgrid-Scale Eddy Viscosity Model, Phys. Fluids 3 (1990) 1760.

[13] M. Germano, Turbulence: The Filtering Approach, J. Fluid Mech. 238 (1992) 325 .

[14] D. Lilly, A Proposed Modification of the Germano Subgrid-Scale Closure Method, Phys. Fluids A 4 (1992) 633.

[15] J. Bardina, J. Ferziger and W. Reynolds, Improved Subgrid Scale Models for Large Eddy Simulation, AIAA Paper 80-1357 (1980).

[16] Y. Zang, R. Street and J. Koseff, A Dynamic Subgrid-Scale Model and Its Application to Turbulent Recirculating Flows, Phys. Fluids A 5 (1993) 3186.

[17] M. Salvetti and S. Banerjee, A Priori Tests of a New Dynamic Subgrid-Scale Model for Finite-Difference Large-Eddy Simulation, Phys. Fluids 7 (1995) 2831.

[18] U. Piomelli, Large-Eddy Simulation: Achievements and Challenges, Prog. Aero. Sci. 35 (1999) 335. 
[19] C. Meneveau and J. Katz, Scale-Invariance and Turbulence Models for LargeEddy Simulation Ann. Rev. Fluid Mech. 32 (2000) 1.

[20] S. Pope, Ten Questions Concerning the Large-Eddy Simulation of Turbulent Flows, New J. Phys. 6 (2004) 35.

[21] B. Galperin and S. Orszag (Eds.), Large Eddy Simulation of Complex Engineering and Geophysical Flows, Cambridge University Press, New York (1993).

[22] P. Sagaut, Large Eddy Simulation of Incompressible Flows, Springer, New York (2003).

[23] S. Chen and G. Doolen, Lattice Boltzmann Method for Fluid Flows, Annu. Rev. Fluid Mech. 8 (1998) 2527.

[24] S. Succi, The Lattice Boltzmann Equation for Fluid Dynamics and Beyond, Clarendon Press, Oxford (2001).

[25] F. J. Higuera, S. Succi and R. Benzi, Lattice gas-dynamics with enhanced collisions, Europhys. Lett. 9 (1989) 345.

[26] X. He and L.S. Luo, A priori derivation of the lattice Boltzmann equation, Phys. Rev. E 55 (1997) 115.

[27] T. Abe, Derivation of the Lattice Boltzmann Method by means of the discrete ordinate method for the Boltzmann equation, J. Comp. Phys. 131 (1997) 241.

[28] L.-S. Luo, Theory of the lattice Boltzmann method: Lattice Boltzmann models for nonideal gases, Phys. Rev. E 62 (2000) 4982.

[29] X. He and G. Doolen, Thermodynamic Foundations of Kinetic Theory and Lattice Boltzmann Models for Multiphase Flows, J. Stat. Phys. 107 (2002) 1572 .

[30] P. Asinari, Semi-Implicit-Linearized Multiple-Relaxation-Time Formulation of Lattice Boltzmann Schemes for Mixture Modeling, Phys. Rev. E 73 (2006) 056705 .

[31] M. Junk, A. Klar and L.S. Luo, Asymptotic Analysis of the Lattice Boltzmann Equation, J. Comp. Phys. 210 (2005) 676.

[32] A.J.C. Ladd and R. Verberg, Lattice-Boltzmann Simulations of Particle-Fluid Suspensions, J. Stat. Phys. 104 (2001) 1191.

[33] S. Succi, I. Karlin and H. Chen, Role of the H theorem in Lattice Boltzmann Hydrodynamic Simulations, Rev. Mod. Phys. 74 (2002) 1203.

[34] D. Yu , R. Mei, L.-S. Luo and W. Shyy, Viscous Flow Computations with the Method of Lattice Boltzmann Equation, Prog. Aero. Sci. 39 (2003) 329.

[35] R.R. Nourgaliev, T.N. Dinh, T.G. Theofanous and D. Joseph, The lattice Boltzmann equation method: Theoretical interpretation, numerics and implications, Int. J. Multiphase Flow 29 (2003) 117. 
[36] H. Chen, S. Succi and S. Orszag, Analysis of Subgrid Scale Turbulence using the Boltzmann Bhatnagar-Gross-Krook Kinetic Equation, Phys. Rev. E 59 (1998) R2527.

[37] S. Ansumali, I. Karlin and S. Succi, Kinetic Theory of Turbulence Modelling: Smallness Parameter, Scaling and Microscopic Derivation of Smagorinsky Model, Physica A 338 (2004) 379.

[38] H. Chen, S. Orszag and I. Staroselsky, Expanded Analogy between Boltzmann Kinetic Theory of Fluids and Turbulence, J. Fluid Mech. 519 (2004) 301.

[39] H. Chen, S. Kandasamy, S. Orszag, R. Shock, S. Succi and V. Yakhot, Extended Boltzmann Kinetic Equation for Turbulent Flows, Science 301 (2003) 633.

[40] D. Martinez, W. Matthaeus, S. Chen and D. Montgomery, Comparison of Spectral Method and Lattice Boltzmann Simulations of Two-dimensional Hydrodynamics, Phys. Fluids 6 (1994) 1285.

[41] G. Amati, S. Succi and R. Benzi, Turbulent Channel Flow Simulations using a Coarse-Grained Extensions of the Lattice Boltzmann Method, Fluid Dyn. Res. 19 (1997) 289.

[42] D. Yu and S. Girimaji, DNS of Homogeneous Shear Turbulence Revisited with the Lattice Boltzmann Method, J. Turb. 6 (2005) 1.

[43] H. Yu, S. Girimaji and L.-S. Luo, Lattice Boltzmann Simulations of Decaying Homogeneous Isotropic Turbulence, Phys. Rev. E 71 (2005) 016708.

[44] P. Lammers, K.N. Beronov, R. Volkert, G. Brenner and F. Durst, Lattice BGK Direct Numerical Simulation of Fully Developed Turbulence in Incompressible Plane Channel Flow, Comput. Fluids 35 (2006) 1137.

[45] J.G.M. Eggels, Direct and Large-Eddy Simulation of Turbulent Fluid Flow using the Lattice-Boltzmann Scheme, Int. J. Heat and Fluid Flow 17 (1996) 307.

[46] S. Hou, J. Sterling, S. Chen and G.D. Doolen, A Lattice Boltzmann Subgrid Model for High Reynolds Number Flows, Fields Inst. Comm. 6 (1996) 151.

[47] Z. Lu, Y. Liao, Y. Qian, J.J. Derksen and K. Kontomaris, Large Eddy Simulations of a Stirred Tank using the Lattice Boltzmann Method on a Nonuniform Grid, J. Comput. Phys. 181 (2002) 675.

[48] M. Krafczyk, J. Tolke and L.-S. Luo, Large-Eddy Simulations with a MultipleRelaxation-Time LBE Model, Int. J. Mod. Phys. B 17 (2003) 33.

[49] H. Yu, S. Girimaji and L.-S. Luo, DNS and LES of Decaying Isotropic Turbulence with and without Rotation using Lattice Boltzmann Method, J. Comput. Phys. 209 (2005) 599.

[50] H. Yu, L.-S. Luo and S. Girimaji, LES of Turbulent Square Jet Flow using an MRT Lattice Boltzmann Model, Comput. Fluids 35 (2006) 957. 
[51] P. Bhatnagar, E. Gross and M. Krook, A Model for Collision Processes in Gases. I. Small Amplitude Processes in Charged and Neutral One-Component Systems, Phys. Rev. 94 (1954) 511.

[52] Y. Qian, D. d'Humières and P. Lallemand, Lattice BGK Models for NavierStokes Equation, Europhys. Lett. 17 (1992) 479.

[53] H. Chen, S. Chen and W.H. Matthaeus, Recover of the Navier-Stokes Eqauations using the Lattice-Gas Boltzmann Method, Phys. Rev. A 45 (1992) 5339 .

[54] D. d'Humières, Generalized Lattice Boltzmann Equations, Progress in Aeronautics and Astronautics (Eds. B.D. Shigal and D.P Weaver) 159 (1992) 450 .

[55] P. Lallemand and Li-Shi Luo, Theory of the Lattice Boltzmann Method: Dispersion, Dissipation, Isotropy, Galilean Invariance, and Stability, Phys. Rev. E 61 (2000) 6546.

[56] D. d'Humières, I. Ginzburg, M. Krafczyk, P. Lallemand and L.-S. Luo, MultipleRelaxation-Time Lattice Boltzmann Models in Three Dimensions, Phil. Trans. R. Soc. Lond. A 360 (2002) 437.

[57] M.E. McCracken and J. Abraham, Multiple-Relaxation-Time LatticeBoltzmann Model for Multiphase Flow, Phys. Rev. E 71 (2005) 036701.

[58] K.N. Premnath and J. Abraham, Three-dimensional Multi-Relaxation Time (MRT) Lattice-Boltzmann Models for Multiphase Flow, J. Comp. Phys. 224 (2007) 539.

[59] K.N. Premnath and J. Abraham, Simulations of Binary Drop Collisions with a Multiple-Relaxation-Time Lattice-Boltzmann Model, Phys. Fluids 17 (2005) 122105 .

[60] M.E. McCracken and J. Abraham, Simulations of Liquid Break Up With an Axisymmetric, Multiple Relaxation Time, Index-Function Lattice Boltzmann Model, Int. J. Mod. Phys. C 16 (2005) 1671.

[61] M.J. Pattison, K.N. Premnath, N.B. Morley and M. Abdou, Progress in Lattice Boltzmann Methods for Magnetohydrodynamic Flows Relevant to Fusion Applications, Fusion Engg. Des. 83 (2008) 557.

[62] K.N. Premnath, M.J. Pattison and S. Banerjee, Generalized Lattice Boltzmann Equation with Forcing Term for Computation of Wall Bounded Turbulent Flows, Phys. Rev. E, submitted (2008).

[63] S. Chapman and T.G. Cowling, Mathematical Theory of Nonuniform Gases, Cambridge University Press, New York (1964).

[64] X. He, L.-S. Luo and M. Dembo, Some Progress in Lattice Boltzmann Method. Part I. Nonuniform Mesh Grids, J. Comput. Phys. 129 (1996) 537. 
[65] H. Chen, O. Filippova, J. Hoch, K. Molving, R. Shock, C. Teixeira and R. Zhang, Grid Refinement in Lattice Boltzmann Methods Based on Volumetric Formulation, Physica A 362 (2006) 158.

[66] M. Rohde, D. Kandhai, J.J. Derksen and H.E.A. van den Akker, A Generic, Mass Conservative Local Grid Refinement Technique for Lattice-Boltzmann Schemes, Int. J. Num. Meth. Fluids 51 (2006) 439.

[67] A. Kravchenko, P. Moin and R. Moser, Zonal Embedded Grids for Numerical Simulations of Wall-Bounded Turbulent Flows, J. Comput. Phys. 127 (1996) 412 .

[68] F. Najjar and D. Tafti, Study of Discrete Test Filters and Finite Difference Approximations for the Dynamic Subgrid-Scale Stress Model, Phys. Fluids 8 (1996) 1076.

[69] J. Kim, P. Moin and R. Moser, Turbulence Statistics in Fully Developed Channel Flow at Low Reynolds Number, J. Fluid Mech. 177 (1987) 133.

[70] K. Lam and S. Banerjee, On the Condition of Streak Formation in a Bounded Turbulent Flow, Phys. Fluids A 4 (1992) 306.

[71] A.J.C. Ladd, Numerical Simulations of Particulate Suspensions via a Discretized Boltzmann Equation. Part 1. Theoretical Foundation, J. Fluid. Mech. 271 (1994) 285.

[72] K. Moin and K. Mahesh, Direct Numerical Simulation: A Tool in Turbulence Research, Ann. Rev. Fluid Mech. 30 (1998) 539.

[73] R. Mei, L.-S. Luo, P. Lallemand and D. d'Humières, Consistent Initial Conditions for Lattice Boltzmann Simulations, Comput. Fluids 35 (2006) 855.

[74] D. Choi, D. Prasad, M. Wang and C. Pierce, Evaluation of an industrial CFD code for LES applications, Proceedings of the Summer Program 2000, Center for Turbulence Research, Stanford, CA (2000).

[75] J. Gullbrand and F.K. Chow, The Effect of Numerical Errors and Turbulence Models in Large-Eddy Simulations of Channel Flow, With and Without Explicit Filtering, J. Fluid Mech. 495 (2003) 323.

[76] H. Kreplin and H. Eckelmann, Behavior of the Three Fluctuating Velocity Components in the Wall Region of a Turbulent Channel Flow, Phys. Fluids 28 (1979) 1233.

[77] R. Moser, J. Kim and N. Mansour, Direct Numerical Simulations of Turbulent Channel Flow Up To $R e_{\tau}=590$, Phys. Fluids 11 (1999) 943. 